\title{
On a theorem of Ranee Brylinski
}

\author{
Bertam Kostant Nolan Wallach
}

January 26, 2009

\section{Introduction}

In her thesis [RB], Ranee Brylinski (then Gupta) studied the orbit structure of the projective variety of abelian subalgebras of a fixed dimension, $k$, in a simple Lie algebra, $\mathfrak{g}$, over $\mathbb{C}$ under its adjoint group, $G$. Fix a Borel subalgebra, $\mathfrak{b}$, of $\mathfrak{g}$ and let $B$ be the closed subgroup of $G$ corresponding to $\mathfrak{b}$. Then the Borel fixed point theorem implies that the closed $G$-orbits are precisely the orbits of the abelian ideals in $\mathfrak{b}$ of dimension $k$. Let $l$ be the rank of $\mathfrak{g}$ and fix $\mathfrak{h}$ a Cartan subalgebra. The main result in $[\mathrm{RB}]$ is that the closed orbits are all contained in the closures of the orbits of the subspaces of $\mathfrak{h}$ of dimension $k$. In particular, if $k=l$ the closure of the orbit of $\mathfrak{h}$ contains all of the closed orbits.

In this paper we will only study the case when $k=l$ (although we expect that similar methods will work for $k<l$ ) and we prove the following strengthening of Brylinski's theorem.

Theorem 1 Let $x$ be a regular (not necessarily semisimple) element of $\mathfrak{g}$ contained in $\mathfrak{b}$. Then the closure of the B-orbit of $\mathfrak{g}^{x}=\{y \in \mathfrak{g} \mid[x, y]=0\}$ contains all of the abelian ideals of $\mathfrak{b}$ of dimension $l$.

Since there is a regular nilpotent element in the closure of the $B$ orbit of each regular element of the projective space on $\mathfrak{g}$, this theorem follows from the special case of $x=e$ a principal (i.e. regular) nilpotent element (see Proposition 3 and its corollary). For this reason we will concentrate on the centralizers of regular nilpotent elements.

In [K3] the first named author proved (in particular) that if we look upon $\wedge^{l} \mathfrak{g}$ as a $\mathfrak{g}$-module in the usual way and if $C$ is the Casimir operator corresponding to the Killing form of $\mathfrak{g}$ then its eigenvalues on $\wedge^{l} \mathfrak{g}$ are all less than or equal to $l$. Let $V_{l}$ be the $l$-eigenspace for $C$ on $\wedge^{l} \mathfrak{g}$. He also proved that relative to the choice of $\mathfrak{b}$ the highest weight spaces of the $l$-eigenspace in $\wedge^{l} \mathfrak{g}$ are exactly the lines $\wedge^{l} \mathfrak{a}$ with $\mathfrak{a}$ an abelian ideal in $\mathfrak{b}$ of dimension $l$. In light of this we have the following result as an immediate implication of the above theorem.

Corollary 2 Let $x \in \mathfrak{g}$ be regular then the $\mathfrak{g}$-cyclic space of $\wedge^{l} \mathfrak{g}^{x}$ in $\wedge^{l} \mathfrak{g}$ is $V_{l}$.

For $x$ regular semisimple this corollary can be found in $[\mathrm{RB}]$. 
Unfortunately, the proof of Theorem 1 is by a case by case check through the classification of simple Lie algebras over $\mathbb{C}$ (as was Brylinski's original argument). The hearty reader will find a few general theorems (e.g. Theorem 7) that do not use the classification that should be much more palatable and demonstrate a beautiful underpinning of the results of this paper.

We dedicate this work to our long time friend Veervalli (Raja) Varadarajan in honor of his seventieth birthday.

\section{Some observations about $\mathfrak{g}^{e}$.}

Let $\mathfrak{g}$ be a semisimple Lie algebra over $\mathbb{C}$ and let $\mathfrak{h}$ be a Cartan subalgebra of $\mathfrak{g}$. Let $\Phi$ denote the root system of $\mathfrak{g}$ with respect to $\mathfrak{h}$, let $\Phi^{+}$be a system of positive roots and let $\Delta$ be the corresponding simple roots. If $\alpha \in \Phi$ then let $\mathfrak{g}_{\alpha}$ denote the corresponding root space. Let $B$ denote the Killing form of $\mathfrak{g}$. We fix a principal nilpotent element $e \in \mathfrak{g}$ which (after possibly acting by $G$ ) we write as

$$
e=\sum_{\alpha \in \Delta} X_{\alpha}
$$

with $X_{\alpha} \neq 0$ for $\alpha \in \Delta$. Let $H \in \mathfrak{h}$ be defined by $\alpha(H)=1$ for all $\alpha \in \Delta$. Then clearly, $[H, e]=e$. If $x \in \mathfrak{g}$ then $x=u+\sum_{\alpha \in \Phi} x_{\alpha}$ with $u \in \mathfrak{h}$ and $x_{\alpha} \in \mathfrak{g}_{\alpha}$. We define

$$
\text { supp } x=\left\{\alpha \in \Phi \mid x_{\alpha} \neq 0\right\} .
$$

Let $G$ denote the adjoint group of $\mathfrak{g}$ (the identity component of the group of automorphisms) and let $\mathcal{O}(\mathfrak{g})$ denote the polynomial functions on $\mathfrak{g}$ and let $\mathcal{O}(\mathfrak{g})^{G}$ denote the set of all $G$-invariant polynomials (i.e. $f(g x)=f(x), x \in \mathfrak{g}$ and $g \in G$ ). If $x \in \mathfrak{g}, f \in \mathcal{O}(\mathfrak{g})$ then we define $\nabla f(x) \in \mathfrak{g}$ by

$$
\frac{d}{d t} f(x+t y)_{\mid t=0}=B(\nabla f(x), y)
$$

for all $y \in \mathfrak{g}$. One checks that if $f \in \mathcal{O}(\mathfrak{g})^{G}$ then

$$
g \nabla f(x)=\nabla f(g x), g \in G, x \in \mathfrak{g} .
$$

Thus in particular, if $f \in \mathcal{O}(\mathfrak{g})^{G}$ then $\nabla f(x) \in \mathfrak{g}^{x}=\{y \in \mathfrak{g} \mid[x, y]=0\}$.

Let $\phi_{1}, \ldots, \phi_{l}$ be a minimal set of homogeneous generators for $\mathcal{O}(\mathfrak{g})^{G}$ ordered by increasing degree. One has $l=\operatorname{dim} \mathfrak{h}$. Chevalley has shown that $\phi_{1}, \ldots, \phi_{l}$ are algebraically independent. Such a (partially) ordered set $\phi_{1}, \ldots, \phi_{l}$ is called a basic set of invariants. The first named author has shown

Theorem 3 Let $x \in \mathfrak{g}$ then $x$ is a regular element in $\mathfrak{g}$ (i.e. $\operatorname{dim} \mathfrak{g}^{x}=\operatorname{dim} \mathfrak{h}$ ) if and only if the elements $\nabla \phi_{1}(x), \ldots, \nabla \phi_{l}(x)$ are linearly independent.

This result implies that for a regular element and a choice of $\phi_{1}, \ldots, \phi_{l}$ the elements $\nabla \phi_{1}(x), \ldots, \nabla \phi_{l}(x)$ form a basis of $\mathfrak{g}^{x}$.

If $V$ is a vector space over $\mathbb{C}$ and if $x \in V$ is nonzero let $[x]$ denote the corresponding point in the projective space, $\mathbb{P}(V)$. 
Proposition 4 If $x \in \mathfrak{b}$ is a regular element of $\mathfrak{g}$ then $[e]$ is contained in $\overline{B[x]}$.

Proof. Let $x=x_{s}+x_{n}$ be the Jordan decomposition of $x$. Then we may assume that $x_{s} \in \mathfrak{h}$ and

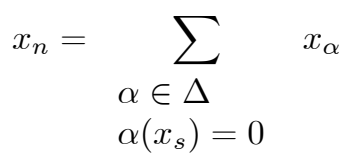

with each of the indicated summands nonzero. Consider the TDS (standard basis of a three dimensional simple Lie algebra) containing to $x_{n}: u, x_{n}, y$ with $u \in \mathfrak{h}$ and $\left[y, x_{s}\right]=0$. Set

$$
\begin{array}{cl}
\mathfrak{v}=\oplus & \alpha \in \Phi^{+} \quad \mathfrak{g}_{\alpha} . \\
& \alpha\left(x_{s}\right) \neq 0
\end{array}
$$

In $[\mathrm{K} 1]$ it is proved that there exists a map $z: \mathbb{C}^{\times} \rightarrow \mathfrak{v}$ such that

$$
e^{a d(z(t))} x_{s}=x_{s}+\frac{1}{t} e^{\prime}
$$

where

$$
e^{\prime}=\sum_{\substack{\alpha \in \Delta \\ \alpha\left(x_{s}\right) \neq 0}} x_{\alpha}
$$

with all the indicated summands nonzero. Thus

$$
e^{a d z(t)} e^{-\log (t) a d u / 2} x=x_{s}+\frac{1}{t} e^{\prime}+\frac{1}{t} e^{a d z(t)} x_{n} .
$$

We write the term $e^{a d z(t)} x_{n}=x_{n}+w(t)$ with $w(t) \in[\mathfrak{n}, \mathfrak{n}]$. Thus if $s(t)=$ $e^{a d z(t)} e^{-\log (t) a d u / 2}$. Then

$$
t s(t) x=t x_{s}+e^{\prime}+x_{n}+w(t) .
$$

This implies that

$$
s(t)[x]=\left[t x_{s}+e^{\prime}+x_{n}+w(t)\right] .
$$

Let $\left\{t_{j}\right\}$ be a sequence in $(0,1)$ converging in $(0,1)$ to 0 . Then by taking a subsequence we may assume that

$$
\lim _{j \rightarrow \infty}\left[t_{j} x_{s}+e^{\prime}+x_{n}+w\left(t_{j}\right)\right]
$$

exists. This limit is of the form $\left[e^{\prime}+x_{n}+w_{o}\right]$ with $w_{o} \in[\mathfrak{n}, \mathfrak{n}] . e^{\prime}+x_{n}+w_{o}$ is a principal nilpotent element in $\mathfrak{n}$ so there exists $b \in B$ such that $b\left(e^{\prime}+x_{n}+w_{o}\right)=$ $e$. This completes the proof.

We note that there is a more direct proof that $\overline{G[x]}$ contains $[e]$. Indeed from [K2] we know that there exists $g \in G$ such that $g x=f+z$ with $z \in \mathfrak{b}$ 
and $f=\sum_{\alpha \in \Delta} x_{-\alpha}$ with $x_{\alpha} \neq 0$ for $\alpha \in \Delta$. We note that $z=\sum_{j \geq 0} z_{j}$ with $\left[H, z_{j}\right]=j z_{j}$. Thus $e^{t a d H} g x=e^{-t} f+\sum_{j \geq 0} e^{j t} z_{j}$. Hence

$$
e^{t} e^{t a d H} g x=f+\sum_{j \geq 0} e^{(j+1) t} z_{j} .
$$

This implies that $\lim _{t \rightarrow-\infty}\left[e^{\operatorname{tadH}} g x\right]=[f]$.

Corollary 5 If $x \in \mathfrak{b}$ is a regular element of $\mathfrak{g}$ then $\mathfrak{g}^{e} \in \overline{B \mathfrak{g}^{x}}$ (here the closure is taken in the Grassmannian of $l$ dimensional subspaces of $\mathfrak{g}$ ).

Proof. We use the standard imbedding of the Grassmannian into the projective space $\mathbb{P}\left(\wedge^{l} \mathfrak{g}\right)$. Then Theorem 3 implies that $\mathfrak{g}^{x}$ corresponds the the line $\left[\nabla \phi_{1}(x) \wedge \cdots \wedge \nabla \phi_{l}(x)\right]$ and so the equivariance assertion above for the maps $\nabla \phi: \mathfrak{g} \rightarrow \mathfrak{g}$ for $\phi \in \mathcal{O}(\mathfrak{g})^{G}$ and the previous result imply this corollary.

If $S \subset \Delta$ then we denote by $\Phi_{S}$ the set $\Phi \cap \sum_{\alpha \in S} \mathbb{Z} \alpha$. Let $\mathfrak{g}_{S}=\mathfrak{h}+\sum_{\alpha \in \Phi_{S}} \mathfrak{g}_{\alpha}$. Then $e_{S}=\sum_{\alpha \in S} X_{\alpha}$ is a principal nilpotent element of $\mathfrak{g}_{S}$.

Lemma 6 Let $f \in \mathcal{O}(\mathfrak{g})^{G}$ be homogeneous of degree $j$ and if $S \subset \Delta$ then $\nabla f\left(e_{S}\right)=(j-1) !(-1)^{j-1} a d\left(e_{S}\right)^{j-1} \nabla f(H)$.

Proof. We have

$$
e^{\operatorname{tad}\left(e_{S}\right)} \nabla f(H)=\nabla f\left(e^{\operatorname{tad}\left(e_{S}\right)} H\right)=\nabla f\left(H-t e_{S}\right)=t^{j-1} \nabla f\left(\frac{1}{t} H-e_{S}\right) .
$$

Thus

$$
t^{1-j} e^{\operatorname{tad}\left(e_{S}\right)} \nabla f(H)=\nabla f\left(\frac{1}{t} H-e_{S}\right)
$$

Now take the limit as $t \rightarrow+\infty$ and the Lemma follows.

Lemma 7 If $f \in \mathcal{O}(\mathfrak{g})^{G}$ is homogeneous and such that $\nabla f(e) \neq 0$ then there exists a basic set of invariants for $G$ containing $f$.

Proof. Let $j$ be the degree of $f$. The content of the assertion is that $f$ is not contained in the subalgebra of $\mathcal{O}(\mathfrak{g})^{G}$ generated by $\sum_{i<j} \mathcal{O}^{i}(\mathfrak{g})^{G}\left(\mathcal{O}^{i}(\mathfrak{g})\right.$ the polynomials of degree $i$ on $\mathfrak{g})$. This is obvious since $h(e)=0$ if $h \in \mathcal{O}^{i}(\mathfrak{g})^{G}$ with $i>0$.

Lemma 8 Let $f \in \mathcal{O}^{i}(\mathfrak{g})^{G}$ with $i>0$. If $\operatorname{supp} \nabla f(e) \cap \Phi_{S} \neq \emptyset$ then $\phi=$ $f_{\left[\left[\mathfrak{g}_{S}, \mathfrak{g}_{S}\right]\right.}$ is contained in a set of basic invariants for $\left[\mathfrak{g}_{S}, \mathfrak{g}_{S}\right]$ and $\operatorname{supp} \nabla \phi\left(e_{S}\right)=$ $\operatorname{supp} \nabla f(e) \cap \Phi_{S}$.

Proof. We note that $\mathfrak{g}=\mathfrak{g}_{S} \oplus V$ with $V=\sum_{\alpha \notin \Phi_{S}} \mathfrak{g}_{\alpha}$. This decomposition is $a d \mathfrak{g}_{S}$-invariant. Let $P$ be the corresponding projection of $\mathfrak{g}$ onto $\mathfrak{g}_{S}$. Then $P \nabla f(e)=\sum_{\alpha \in \operatorname{supp} \nabla f(e) \cap \Phi_{S}} \nabla f(e)_{\alpha} \neq 0$. Up to non-zero scalar multiple $c_{i}=$ $\frac{(-1)^{i-1}}{(i-1) !}$

$$
\nabla f(e)=c_{i} a d(e)^{i-1} \nabla f(H)=c_{i} a d\left(e_{S}+e_{\Delta-S}\right)^{i-1} \nabla f(H)
$$


which is equal to $a d\left(e_{S}\right)^{i-1} \nabla f(H)$ plus a sum of terms that are multiples of expressions of the form $u_{1} \cdots u_{i-1} \nabla f(H)$ with at least one $u_{i}=\operatorname{ad}\left(e_{\Delta-S}\right)$ and the other $u_{j}$ either $a d\left(e_{S}\right)$ or $a d\left(e_{\Delta-S}\right)$. Hence we see that $P u_{1} \cdots u_{i-1} \nabla f(H)=$ 0 for each of those terms. Thus $0 \neq P \nabla f(e)=c_{i} a d\left(e_{S}\right)^{i-1} \nabla f(H)=\nabla f\left(e_{S}\right)=$ $\nabla f_{\mid\left[\mathfrak{g}_{S}, \mathfrak{g}_{S}\right]}\left(e_{S}\right)$. The result now follows from the previous lemma.

We are now ready for our first result about supports.

Theorem 9 If $\mathfrak{g}$ is simple and not of type $D_{l}, E_{6}$,or $E_{7}$ then

$$
\operatorname{supp}\left(\nabla \phi_{i}(e)\right)=\left\{\alpha \in \Phi^{+} \mid \alpha(H)=\operatorname{deg} \phi_{i}-1\right\} .
$$

After we prove this result we will look at the corresponding result for $\mathrm{D}_{l}, \mathrm{E}_{6}$ and $\mathrm{E}_{7}$ in each case we will show that there is one exceptional $i$. We set $\mathcal{O}(\mathfrak{g})_{+}^{G}=$ $\left\{f \in \mathcal{O}(\mathfrak{g})^{G} \mid f(0)=0\right\}$. Since $e \in \mathcal{N}=\left\{x \in \mathfrak{g} \mid \phi(x)=0, \phi \in \mathcal{O}(\mathfrak{g})_{+}^{G}\right\}$ we see that if $\phi, \psi \in \mathcal{O}(\mathfrak{g})^{G}$ and if $\phi-\psi \in\left(\mathcal{O}(\mathfrak{g})_{+}^{G}\right)^{2}$ then $\nabla \phi(e)=\nabla \psi(e)$. Thus in light of Chevalley's theorem and the fact that under our hypotheses $\operatorname{deg} \phi_{i} \neq \operatorname{deg} \phi_{j}$ for $i \neq j$ we need only prove the result for one choice of $\phi_{1}, \ldots, \phi_{l}$. We will prove the theorem by a case by case check.

$\mathrm{A}_{n}$ : We realize $\mathfrak{g}$ (as usual) as $\left\{x \in M_{n+1}(\mathbb{C}) \mid \operatorname{tr} X=0\right\}$. Then we may take $e=\sum_{i=1}^{n} E_{i i+1}$ (one nilpotent Jordan block). We take $\phi_{i}(x)=\operatorname{tr}\left(x^{i}\right)$. Thus (up to scalar multiple) $\nabla \phi_{i}(x)=x^{i-1}$. If we use the standard " $\varepsilon$ " notation for the roots then if $j$ is given then the set $\left\{\alpha \in \Phi^{+} \mid \alpha(H)=j\right\}$ is 0 if $j>n$ and is equal to $\left\{\varepsilon_{i}-\varepsilon_{i+j} \mid i=1, \ldots, n+1-j\right\}$. Obviously,

$$
e^{j}=\sum_{i=1}^{n+1-j} E_{i i+j} .
$$

This is the content of the theorem for $A_{n}$.

For the $\mathrm{B}_{n}$ and $\mathrm{C}_{n}$ case we will introduce some notation. We take $L_{n}$ to be the skew diagonal $n \times n$ matrix with ones on the main skew diagonal. That is,

$$
L_{5}=\left[\begin{array}{lllll}
0 & 0 & 0 & 0 & 1 \\
0 & 0 & 0 & 1 & 0 \\
0 & 0 & 1 & 0 & 0 \\
0 & 1 & 0 & 0 & 0 \\
1 & 0 & 0 & 0 & 0
\end{array}\right]
$$

We realize $\mathrm{B}_{n}$ as the elements $x \in M_{2 n+1}(\mathbb{C})$ such that $\left(x^{T}\right.$ is the transpose of $x)$

$$
x L_{2 n+1}+L_{2 n+1} x^{T}=0 .
$$

We set $J_{n}$ equal to the $2 n \times 2 n$ block matrix

$$
\left[\begin{array}{cc}
0 & L_{n} \\
-L_{n} & 0
\end{array}\right]
$$


and we realize $\mathrm{C}_{n}$ as the elements $x \in M_{2 n}(\mathbb{C})$ such that

$$
x J_{n}+J_{n} x^{T}=0 .
$$

With this realization of $\mathfrak{g}=\mathrm{B}_{n}$ or $\mathrm{C}_{n}$ we choose $\mathfrak{b}$ to be the upper triangular elements of $\mathfrak{g}$ and $\mathfrak{h}$ to be the diagonal elements. We note that if $x$ is a non-zero nilpotent element of $\mathfrak{u}=\mathfrak{s l}(2, \mathbb{C})$ and if $\left(\sigma_{m}, \mathbb{C}^{m}\right)$ is (up to equivalence) the unique $m$-dimensional irreducible representation of $\mathfrak{u}$ then $\sigma_{m}(x)$ is conjugate to a single nilpotent Jordan block hence defines a principle nilpotent element of $\mathfrak{s l}(m, \mathbb{C})$. Also the representation $\sigma_{m}$ is orthogonal (has a nondegenerate invariant symmetric form) if $m$ is odd, $m=2 n+1$ and is symplectic (has a nondegenerate skew symmetric form) if $m$ is even, $m=2 n$. This implies that for the cases $B_{n}$ or $C_{n}$ a principal nilpotent element is principal nilpotent for the ambient $\mathfrak{s l}(m, \mathbb{C})(m$ respectively, $2 n+1,2 n)$ in our realization. Since we can take $\phi_{i}(v)=\operatorname{tr}\left(v^{2 i}\right)$ in both of these cases. Hence $\nabla \phi_{j}(v)=2 j v^{2 j-1}$. We see that the assertion for these two cases follows from that for the corresponding $\mathrm{A}_{m-1}$.

In type $\mathrm{G}_{2}$ there is nothing to prove since $l=2$ and the assertion of the theorem is true for the invariant of degree 2 (yielding $e$ ) and the basic invariant of a highest degree (yielding the highest root).

For the other exceptional cases we will use results in [K1] and [K2] to prove the following theorem that simplifies the problem. Recall that the Coxeter number of $\mathfrak{g}$ is $h=\operatorname{deg} \phi_{l}$.

Theorem 10 If the number $\operatorname{deg} \phi_{i}-1$ is relatively prime to $h$ then

$$
\operatorname{supp}\left(\nabla \phi_{i}(e)\right)=\left\{\alpha \in \Phi^{+} \mid \alpha(H)=\operatorname{deg} \phi_{i}-1\right\} .
$$

Proof. Set $G$ equal to the adjoint group of $\mathfrak{g}$. Let $\widehat{\Delta}=\Delta \cup\{-\beta\}$ with $\beta$ the highest root in $\Phi^{+}$. We set

$$
z_{o}=\sum_{\alpha \in \widehat{\Delta}} x_{\alpha}=e+x_{-\beta}
$$

with $x_{\alpha} \in \mathfrak{g}_{\alpha}$ for $\alpha \in \widehat{\Delta}$ and $x_{\alpha} \neq 0$. We note that $z_{o}$ is a cyclic element of $\mathfrak{g}$ in the sense of [K1] and [K2] hence in particular it is a regular semisimple element of $\mathfrak{g}$ and hence

$$
\mathfrak{u}=\left\{x \in \mathfrak{g} \mid\left[x, z_{o}\right]=0\right\}
$$

is a Cartan subalgebra. We define $x_{o} \in \mathfrak{h}$ by $\alpha\left(x_{o}\right)=1$ for all $\alpha \in \Delta$. We put

$$
A=\exp \left(2 \pi i a d\left(x_{o}\right) / h\right) \in G
$$

Then if $c=e^{2 \pi i / h}$ then since $\beta\left(x_{o}\right)=h-1, A z_{o}=c z_{o}$. We also observe that the order of $A$ as $h$ and that $A$ is a regular semisimple element of $G$ hence it is a principal element in the sense of [K1]. In [K1] it is shown that the set 
of principal elements of $G$ form one conjugacy class and since it has order $h$ on $\mathfrak{u}$ we see that $A_{\mid \mathfrak{u}}$ defines a Coxeter element of the Weyl group of $\mathfrak{u}$. Let $\phi_{1}, \ldots, \phi_{l}$ be a set of homogeneous fundamental $G$ invariant polynomials on $\mathfrak{g}$ with $\operatorname{deg} \phi_{i} \leq \operatorname{deg} \phi_{i+1}$. Since $z_{o}$ is regular the elements $u_{i}=\nabla \phi_{i}\left(z_{o}\right)$ are linearly independent. Set $m_{i}=\operatorname{deg} \phi_{i}-1$. Observe that $m_{l}=h-1$. Also

$$
A u_{i}=A \nabla \phi_{i}\left(z_{o}\right)=\nabla \phi_{i}\left(A z_{o}\right)=\nabla \phi_{i}\left(c z_{o}\right)=c^{m_{i}} u_{i}
$$

In particular this implies that

$$
u_{i}=v_{i}+y_{i}
$$

with $a d x_{o} v_{i}=m_{i} v_{i}$ and $a d x_{o} y_{i}=\left(m_{i}-h\right) y_{i}$. We note that

$$
e^{t a d x_{o}} \nabla \phi_{i}\left(z_{o}\right)=\nabla \phi_{i}\left(e^{t a d x_{o}}\left(e+x_{-\beta}\right)\right)=e^{t m_{i}} \nabla \phi_{i}\left(e+e^{-t h} x_{-\beta}\right) .
$$

We therefore have

$$
v_{i}=\lim _{t \rightarrow+\infty} e^{-t m_{i}} e^{t a d x_{o}} u_{i}=\nabla \phi_{i}(e)
$$

If $\operatorname{gcd}\left(m_{i}, h\right)=1$ then let $1 \leq q_{i} \leq h-1$ be defined by $q_{i} m_{i} \equiv 1 \bmod h$. Then $A^{q_{i}}$ has order $h$ and is also regular and so it is principle and its restriction to $\mathfrak{u}$ is also a Coxeter element. This implies that there exists $w_{i}$ in the normalizer of $\mathfrak{u}$ in $G$ such that

$$
w_{i} A w_{i}^{-1}=A^{q_{i}} .
$$

Since $A^{q_{i}}$ is a regular element in the Cartan subgroup of $G$ corresponding to $\mathfrak{h}$ we not that $w_{i \mid \mathfrak{h}}$ defines an element of the Weyl group of $\mathfrak{h}$. We note that the above conjugacy implies that $A^{q_{i}} w_{i} z_{o}=c w_{i} z_{o}$. Hence $A w_{i} z_{o}=c^{m_{i}} w_{i} z_{o}$. Hence

$$
w_{i} \widehat{\Delta}=\left\{\alpha \in \Phi \mid \alpha\left(x_{o}\right)=m_{i}\right\} \cup\left\{\alpha \in \Phi \mid \alpha\left(x_{o}\right)=m_{i}-h\right\} .
$$

We therefore have

$$
v_{i}=\sum_{\substack{\alpha \in \Phi \\ \alpha\left(x_{o}\right)=m_{i}}} x_{\alpha}
$$

with $x_{\alpha} \neq 0$ for all the indices in the sum. This proves the theorem.

This result implies Theorem 9 for $\mathrm{G}_{2}, \mathrm{~F}_{4}$ and $\mathrm{E}_{8}$ and hence completes its proof.

We now determine the supports for the groups not covered by the theorem.

$\mathrm{E}_{6}$ in this case the numbers $\operatorname{deg} \phi_{i}-1$ are respectively $1,4,5,7,8,11$ and $\operatorname{deg} \phi_{6}=12$. So we need only check the support of $\nabla \phi_{i}$ for $i=2,5$. For $i=5$ the set $\left\{\alpha \in \Phi^{+} \mid \alpha(H)=8\right\}$ consists of two roots that are conjugate under the outer involution. If $i=2$ we argue indirectly as follows. We note that we have an $\mathfrak{s l}_{2}$-triple $e, 2 H, f$. Also, up to scalar multiple we have

$$
a d(e)^{4} \nabla \phi_{2}(H)=\nabla \phi_{2}(e) .
$$


We can choose $e$ such that it is invariant under the outer automorphism, $\theta$, gotten from the involution of the Dynkin diagram. We also know that the eigenspace 1 for $\theta$ is a $\mathrm{C}_{4}$ and since $\mathrm{C}_{4}$ has no invariants of degree 5 we see that the restriction of $\phi_{2}$ to $\mathfrak{h}^{\theta}$ is 0 . This implies that $\theta \nabla \phi_{2}(H)=-\nabla \phi_{2}(H)$. Since $\theta e=e$ we see that

$$
\theta \nabla \phi_{2}(e)=-\nabla \phi_{2}(e) .
$$

The set $\Phi_{4}^{+}=\left\{\alpha \in \Phi^{+} \mid \alpha(H)=4\right\}$ consists of (in Bourbaki notation):

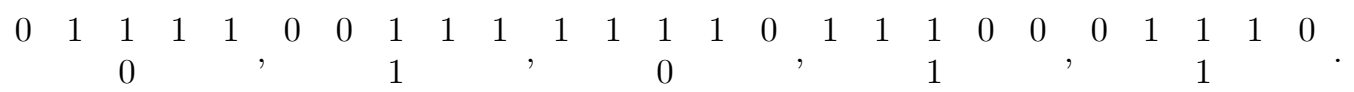

The support of $\nabla \phi_{2}(e)$ is this set with the last element deleted. We will complete the proof of this assertion when we finish our study of the case $\mathrm{D}_{n}$.

$E_{7}$ : In this case we have $\operatorname{deg} \phi_{7}=18$ and the numbers $\operatorname{deg} \phi_{i}-1$ are respectively $1,5,7,9,11,13,17$. Thus the only case not covered by Theorem 9 is $i=4$ $\left(m_{4}=9\right)$. In this case the set $\Phi_{9}^{+}=\left\{\alpha \in \Phi^{+} \mid \alpha(H)=9\right\}$ is

$$
\begin{array}{llllllllllllllllllllllll}
0 & 1 & 2 & 2 & 2 & 1 & 1 & 1 & 2 & 2 & 1 & 1 & 1 & 2 & 2 & 1 & 1 & 1 & 1 & 2 & 2 & 2 & 1 & 0
\end{array} .
$$

Let us choose a root vector $X_{i} \neq 0$ for the $i$-th root above $(i=1, \ldots, 4)$. We note that if $S=\Delta-\left\{\alpha_{7}\right\}$ then $\left[\mathfrak{g}_{S}, \mathfrak{g}_{S}\right]$ is isomorphic with $\mathrm{E}_{6}$. Since $\mathrm{E}_{6}$ doesn't have a basic invariant of degree 10 we see that the last root cannot be in the support (Lemma 8). If we calculate with $S=\Delta-\left\{\alpha_{1}\right\}$ then we find by a calculation for $\mathrm{D}_{6}$ that $\nabla \phi_{4}\left(e_{S}\right) \neq 0$ so the first root is in the support. We also note that $\Phi_{10}^{+}$consists of

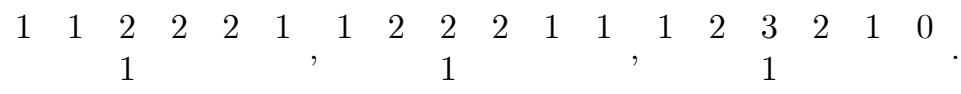

We take $e=e_{1}+e_{2}+e_{3}+e_{4}+e_{5}+e_{6}+e_{7}$ with $e_{i} \neq 0$ in the root space for the $i$-th simple root in the Bourbaki order. Let $X=a_{1} X_{1}+a_{2} X_{2}+a_{3} X_{3}+a_{4} X_{4}$. Then using the list of elements in $\Phi_{10}^{+}$we see that $[e, X]$ is given by

$$
a_{1}\left[e_{1}, X_{1}\right]+a_{2}\left[e_{3}, X_{2}\right]+a_{2}\left[e_{6}, X_{2}\right]+a_{3}\left[e_{5}, X_{3}\right]+a_{4}\left[e_{4}, X_{4}\right]+a_{4}\left[e_{7}, X_{4}\right] .
$$

We are solving for $a_{1}, a_{2}, a_{3}, a_{4}$ so that In this expression the only term in

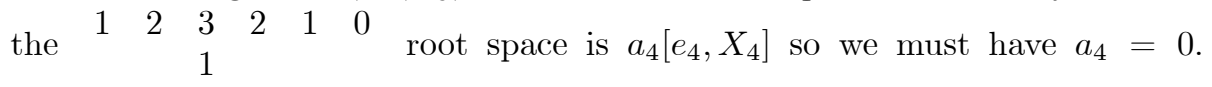
(We have already seen this above.) Since $a_{1} \neq 0$ and since $\left[e_{1}, X_{1}\right]$ is in the $\begin{array}{lllllll}1 & 1 & 2 & 2 & 2 & 1 & \text { root space and the only other term in that root space is }\end{array}$ $\left[e_{6}, X_{2}\right]$ we see that $a_{2} \neq 0$. But $\left[e_{3}, X_{2}\right]$ is in the $\begin{array}{rrrrrrr}1 & 2 & 2 & 2 & 1 & 1 \\ & & & & & & \text { root space }\end{array}$ as is $\left[e_{5}, X_{3}\right]$ thus we must have $a_{3} \neq 0$. 
$\mathrm{D}_{n}$ : We realize $\mathrm{D}_{n}$ as the Lie algebra $\mathfrak{g}=\left\{X \in M_{2 n}(\mathbb{C}) \mid X L_{2 n}+L_{2 n} X^{T}=\right.$ $0\}$. We take $\mathfrak{h}$ to be the diagonal elements in $\mathfrak{g}$ that is the elements

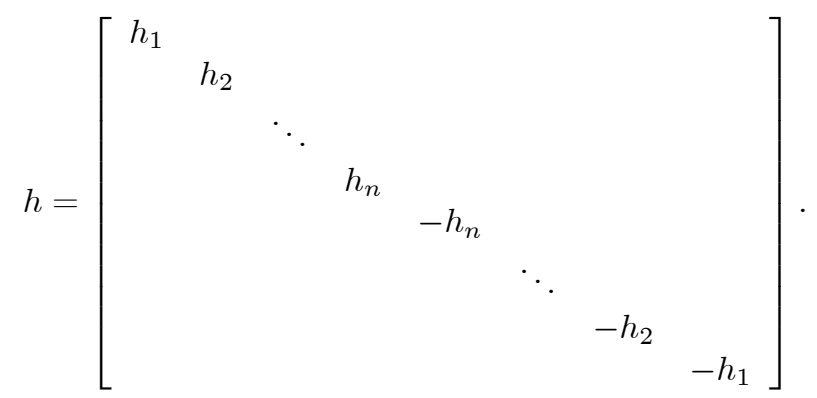

The element $H$ then is given by $h_{i}=n-i$. Also we can take the element $e$ to be

$$
e=\left[\begin{array}{cccccccc}
0 & 1 & & & & & & \\
& 0 & 1 & & & & & \\
& & \ddots & 1 & 1 & & & \\
& & & 0 & 0 & -1 & & \\
& & & & 0 & -1 & & \\
& & & & & \ddots & \ddots & \\
& & & & & & 0 & -1 \\
& & & & & & & 0
\end{array}\right]
$$

that is $\sum_{i=1}^{n-1}\left(E_{i, i+1}-E_{n+i, n+i+1}\right)+E_{n-1, n+1}-E_{n, n+2}$. We take as the invariants $\nu_{i}(X)=\operatorname{tr} X^{2 i}$ for $i=1,2, \ldots, n-1$ and $\nu_{n}(X)=\operatorname{pfaff}(X)$. A direct calculation shows that $\operatorname{supp} \nabla \nu_{i}(e)=S_{2 i-1}=\left\{\alpha \in \Phi^{+} \mid \alpha(H)=2 i-1\right\}$, $1 \leq i \leq n-1$.

Lemma $11 \operatorname{supp} \nabla p f a f f(e)=\left\{\varepsilon_{1}+\varepsilon_{n}, \varepsilon_{1}-\varepsilon_{n}\right\}$.

Proof. We will show that (the blanks should be filled with zeros)

$$
\nabla \operatorname{pfaff}(e)=c_{n}\left[\begin{array}{cccccccc}
0 & 0 & \cdots & 1 & -1 & & & \\
& 0 & \cdots & 0 & 0 & & & \\
& & \ddots & \vdots & \vdots & & & \\
& & & 0 & 0 & 0 & \cdots & 1 \\
& & & & 0 & 0 & & -1 \\
& & & & & \ddots & \ddots & \\
& & & & & & 0 & 0 \\
& & & & & & & 0
\end{array}\right]
$$

with $c_{n} \neq 0$. Indeed, in this case we take $\sigma$ to be the permutation matrix corresponding to the transposition $n \leftrightarrow n+1$. Then

$$
\zeta: x \longmapsto \sigma x \sigma
$$


defines an outer automorphism of $\mathfrak{g}$. One checks that if $\alpha \in \Phi_{n-1}^{+}-\left\{\varepsilon_{1}+\right.$ $\left.\varepsilon_{n}, \varepsilon_{1}-\varepsilon_{n}\right\}$ then $\zeta_{\mid \mathfrak{g}_{\alpha}}$ is multiplication by 1 and $\zeta$ interchanges the root spaces corresponding to $\varepsilon_{1}+\varepsilon_{n}$ and $\varepsilon_{1}-\varepsilon_{n}$. Also on $\mathfrak{h}, \zeta$ acts by $h_{i} \longmapsto h_{i}$ for $i<n$ and $h_{n} \longmapsto-h_{n}$. This implies that

$$
\operatorname{pfaff}(\zeta X)=-\operatorname{pfaff}(X) .
$$

Now $e=\zeta e$. Thus $\zeta \nabla \operatorname{pfaff}(e)=-\nabla \operatorname{pfaff}(e)$. The result now follows since $\nabla \operatorname{pfaff}(e) \neq 0$.

We will now finish the discussion of $\mathrm{E}_{6}$. We note that the Lie subalgebra that corresponds to the roots that have coefficient 0 with respect to the first simple root is the Levi factor $\mathbb{C} \oplus \mathrm{D}_{5}$. This subalgebra contains the Cartan subalgebra. One checks that the restriction of $\phi_{2}$ to this $\mathrm{D}_{5}$ is the Pfaffian. The projection of the $\mathrm{E}_{6}$ " $H$ " into this $\mathrm{D}_{5}$ is the corresponding " $H$ ". This implies that the projection of $\nabla \phi_{2}(H)$ into the $\mathrm{D}_{5}$ is a nonzero scalar multiple of $\nabla \operatorname{pfaff}(H)$. Since the $e$ for $\mathrm{E}_{6}$ is $E_{\alpha_{1}}$ plus the $e$ for $\mathrm{D}_{5}$ we see that in $\operatorname{ad}(e)^{4} \nabla \phi_{2}(H)$ the terms that don't involve the first simple root are the ones that appear for $\mathrm{D}_{5}$ and the Pfaffian. This implies that in the list above the first 2 roots are in the support. The next two are the images of the first two under the outer automorphism. This completes the discussion for $\mathrm{E}_{6}$.

\section{Ideals in $\mathfrak{b}$ of dimension $l$.}

In this section we will give a complete description of these ideals for simple Lie algebras over $\mathbb{C}$. Much of this section can be considered to be a review of results in the MIT thesis of Ranee Brylinski (nee Gupta) [RB]. We maintain the notation of the previous section. We note that since $\mathfrak{h} \subset \mathfrak{b}$ if $\mathfrak{a}$ is an ideal in $\mathfrak{b}$ then $\mathfrak{a}=\mathfrak{a} \cap \mathfrak{h} \oplus \sum_{\alpha \in \Phi^{+}} \mathfrak{g}_{\alpha} \cap \mathfrak{a}$. It is easy to see that if $\operatorname{dim} \mathfrak{a}=l$ then $\mathfrak{a} \cap \mathfrak{h}=\{0\}$. This implies that $\mathfrak{a}$ is determined by the roots $\Phi(\mathfrak{a})=\left\{\alpha \mid \mathfrak{g}_{\alpha} \cap \mathfrak{a} \neq\{0\}\right\}$. Further a subset $\Lambda \subset \Phi^{+}$is a set $\Phi(\mathfrak{a})$ for $\mathfrak{a}$ an ideal in $\mathfrak{b}$ of dimension $l$ if and only if it satisfies the following two conditions

1. $|\Lambda|=l$.

2. If $\alpha \in \Phi^{+}, \beta \in \Lambda$ and if $\alpha+\beta \in \Phi$ then $\alpha+\beta \in \Lambda$.

Fix $\Lambda$ satisfying these two conditions. We note that this implies that if $\psi$ is the highest root then $\psi \in \Lambda$. Also since $\psi(H)=h-1$, we see that if $\alpha \in \Lambda$ then $\alpha(H) \geq h-l$. One can check that 1 . and 2. imply that if $\alpha, \beta \in \Lambda$ then if $\mathfrak{g}$ is not of type A or D then $\alpha(H)+\beta(H) \geq 2 h-2 l \geq h$ so the corresponding $\mathfrak{a}$ is abelian. For type $A_{n}$ and $D_{n}$ we will see that the ideals in $\mathfrak{b}$ of dimension $l$ are abelian as part of our case by case study. We will state this as a lemma and indicate below when the proof is complete.

Lemma 12 If $\mathfrak{g}$ is simple and if $\mathfrak{a}$ is an ideal in $\mathfrak{b}$ with $\operatorname{dim} \mathfrak{a}=l$ then $\mathfrak{a}$ is abelian.

We will now give a (case by case) classification of the sets $\Lambda$. 
$\mathrm{A}_{n}$ : In this case $\Phi^{+}$is the set of all roots $\alpha_{i, j}=\sum_{k=i}^{j} \alpha_{i}$ with $\alpha_{1}, \ldots, \alpha_{n}$ the simple roots in the usual order and $i \leq j$. Clearly, if $\alpha_{i, j} \in \Lambda$ then $\alpha_{r, s} \in \Lambda$ for $1 \leq r \leq i \leq j \leq n$. From this we see that $\Lambda$ corresponds to an ideal of $\mathfrak{b}$ contained in $\mathfrak{n}$ if and only if it is of the form

$\left\{\alpha_{1, m_{1}}, \alpha_{1, m_{1}+1}, \ldots, \alpha_{1, n}\right\} \cup\left\{\alpha_{2, m_{2}}, \alpha_{2, m_{2}+1}, \ldots, \alpha_{2, n}\right\} \cup \cdots \cup\left\{\alpha_{k, m_{k}}, \alpha_{k, m_{k}+1}, \ldots, \alpha_{k, n}\right\}$

with $m_{1} \leq m_{2} \leq \ldots \leq m_{k}$. If $\operatorname{dim} \mathfrak{a}=n$ then we must have $\sum\left(n-m_{i}+1\right)=n$. Thus

$$
n_{1}=n-m_{1}+1 \geq n_{2}=n-m_{2}+1 \geq \ldots \geq n_{k}=n-m_{k}+1>0
$$

defines a partition of $n$. We assert that under this condition $m_{1} \geq k$. Indeed, if $m_{1}<k$ then $n_{1}>n-k+1$. Since $n_{i} \geq 1$, for $i=1, \ldots, k$ we would have $n=\sum n_{i}>n-k+1+(k-1)=n$. 1.,2. and the dimension condition imply that the ideal is abelian in this case. This also implies, in this case, that the number of ideals in $\mathfrak{b}$ of dimension $n$ is $p(n)$ the partition function of $n$. In the " $\varepsilon$ " notation for roots the $\Lambda$ as above are the sets:

$$
\begin{gathered}
\left\{\varepsilon_{1}-\varepsilon_{n-n_{1}+2}, \varepsilon_{1}-\varepsilon_{n-n_{1}+3}, \ldots, \varepsilon_{1}-\varepsilon_{n+1}\right\} \cup \\
\left\{\varepsilon_{2}-\varepsilon_{n-n_{2}+2}, \varepsilon_{2}-\varepsilon_{n-n_{2}+3}, \ldots, \varepsilon_{2}-\varepsilon_{n+1}\right\} \cup \cdots \cup \\
\left\{\varepsilon_{k}-\varepsilon_{n-n_{k}+2}, \varepsilon_{1}-\varepsilon_{n-n_{k}+3}, \ldots, \varepsilon_{k}-\varepsilon_{n+1}\right\}
\end{gathered}
$$

with $n_{1} \geq \ldots \geq n_{k}>0$ and $\sum n_{i}=n$.

$\mathrm{B}_{n}$ : We take $\Delta$ to be $\left\{\alpha_{1}, \ldots, \alpha_{n}\right\}$ in the usual order. Then the positive roots are given by $\alpha_{i, j}=\sum_{k=i}^{j} \alpha_{i}$ for $i \leq j$ and

$$
\beta_{i, j}=\sum_{k=i}^{j} \alpha_{k}+2 \alpha_{j+1, n}, 1 \leq i \leq j<n .
$$

We note that $h=2 n$ so if $\Lambda$ satisfies 1 . and 2. above then $\alpha(H) \geq n$ for all $\alpha \in \Lambda$. Since $\alpha_{i, j}(H)=j+1-i$ we see that the only possible element of the form $\alpha_{i, j}$ that can be an element of $\Lambda$ is $\alpha_{1, n}=\alpha_{1}+\ldots+\alpha_{n}$. If $\Lambda$ contains this element then it must contain $\beta_{1, j}$ for $j=1, \ldots, n-1$ by condition 2 . One checks that the set

$$
\left\{\alpha_{1, n}\right\} \cup\left\{\beta_{1, j} \mid j=1, \ldots, n-1\right\}
$$

satisfies 1 . and 2. We may thus assume that all elements of $\Lambda$ are of the second form. Here we note that if $\beta_{i, j} \in \Lambda$ then $\beta_{p, q} \in \Lambda$ for all $1 \leq p \leq i \leq q \leq j$ this implies that the other $\Lambda$ are of the form

$$
\left\{\beta_{1,1}, \beta_{1,2}, \ldots, \beta_{1, m_{1}}\right\} \cup\left\{\beta_{2,2}, \ldots, \beta_{2, m_{2}}\right\} \cup\left\{\beta_{k, k}, \beta_{k, k+1}, \ldots, \beta_{k, m_{k}}\right\}
$$

with $m_{i} \geq i$ for $i=1, \ldots, k$ and $m_{1} \geq m_{2} \geq \ldots \geq m_{k}$ with $\sum\left(m_{i}-i+1\right)=n$. It is, perhaps, clearer to describe these sets in " $\varepsilon$ " notation. The first set described above $\left(\left\{\alpha_{1, n}\right\} \cup\left\{\beta_{1, j} \mid j=1, \ldots, n-1\right\}\right)$ is

$$
\left\{\varepsilon_{1}+\varepsilon_{2}, \varepsilon_{1}+\varepsilon_{3}, \ldots, \varepsilon_{1}+\varepsilon_{n}, \varepsilon_{1}\right\} .
$$


The set for $m_{1}, \ldots, m_{k}$ is given by

$$
\left\{\varepsilon_{1}+\varepsilon_{2}, \ldots, \varepsilon_{1}+\varepsilon_{m_{1}+1}\right\} \cup\left\{\varepsilon_{2}+\varepsilon_{3}, \ldots, \varepsilon_{2}+\varepsilon_{m_{2}+1}\right\} \cup \cdots \cup\left\{\varepsilon_{k}+\varepsilon_{k+1}, \ldots, \varepsilon_{k}+\varepsilon_{m_{k}+1}\right\} .
$$

Thus the number of such $\Lambda$ in this case is the number of partitions of $n$ with distinct parts. We note that as a consequence of Euler's identity the number of partitions of $n$ with distinct parts is the same as the number of partitions, $p_{o}(n)$, of $n$ with an odd parts (c.f. [A] a discussion of the asymptotics can also be found therein which imply $\left.\sim C_{1} e^{C_{2} \sqrt{n}}\right)$.

$\mathrm{C}_{n}$ : This case is very similar to the previous one. We use the standard labeling of the simple roots. This time the positive roots are in the form $\alpha_{i, j}$, $1 \leq i, j \leq n$ as before and $\beta_{i, j} 1 \leq i \leq j<n$ and

$$
\beta_{i, j}=\sum_{k=i}^{j-1} \alpha_{k}+2 \sum_{k=j}^{n-1} \alpha_{k}+\alpha_{n} .
$$

Assume that $\Lambda$ satisfies 1. and 2. As before, if $\alpha \in \Lambda$ then $\alpha(H) \geq n$. Thus the only element of the form $\alpha_{i j}$ that can be in $\Lambda$ is $\alpha_{1, n}$. The only $\Lambda$ satisfying 1 . and 2. that can contain $\alpha_{1, n}$ is

$$
\left\{\alpha_{1, n}, \beta_{1, n-1}, \beta_{1, n-2}, \ldots, \beta_{1,1}\right\} .
$$

The other elements are of the form

$$
\left\{\beta_{1,1}, \beta_{1,2}, \ldots, \beta_{1, m_{1}}\right\} \cup\left\{\beta_{2,2}, \ldots, \beta_{2, m_{2}}\right\} \cup\left\{\beta_{k, k}, \beta_{k, k+1}, \ldots, \beta_{k, m_{k}}\right\}
$$

with $m_{1} \geq m_{2} \geq \ldots \geq m_{k}$ and $m_{i} \geq i$ and $\sum\left(m_{i}-i+1\right)=n$. We will write these sets in " $\varepsilon$ " notation. The first is

$$
\left\{\varepsilon_{1}+\varepsilon_{n}, \varepsilon_{1}+\varepsilon_{n-1}, \ldots, \varepsilon_{1}+\varepsilon_{2}, 2 \varepsilon_{1}\right\}
$$

the others are

$\left\{\varepsilon_{1}+\varepsilon_{m_{1}}, \ldots, \varepsilon_{1}+\varepsilon_{2}, 2 \varepsilon_{1}\right\} \cup\left\{\varepsilon_{2}+\varepsilon_{m_{2}}, \ldots, \varepsilon_{2}+\varepsilon_{3}, 2 \varepsilon_{2}\right\} \cup \cdots \cup\left\{\varepsilon_{k}+\varepsilon_{m_{k}}, \ldots, \varepsilon_{k}+\varepsilon_{k+1}, 2 \varepsilon_{k}\right\}$.

These all clearly satisfy the conditions 1.,2. Thus number of such ideals in this case is also equal to the number of partitions of $n$ with distinct parts.

$\mathrm{D}_{n}$ : We will only consider the case $n \geq 4$. We note that $h=2 n-2$. Let $\Lambda$ satisfy 1 . and 2. If $\alpha \in \Lambda$ then the argument above implies that $\alpha(H) \geq n-2$. If $\alpha(H)=n-2$ and if there exist two simple roots $\gamma, \delta$ such that $\alpha+\gamma$ and $\alpha+\delta$ are roots then $\alpha$ cannot be a member of $\Lambda$ (since then $|\Lambda| \geq n+1)$. If $n$ is even then every element at this level has this property so if $n$ is even and if $\alpha \in \Lambda$ then $\alpha(H) \geq n-1$. If $n$ is odd consider the roots at level $n-2$. For $n=2 k+1$ the only $\alpha$ at this level such that we can add exactly one simple root to get a root is

$$
\alpha=\alpha_{k+1}+2 \alpha_{k+2}+\ldots+2 \alpha_{2 k-1}+\alpha_{2 k}+\alpha_{2 k+1} .
$$


To this root we can only add $\alpha_{k}$. However to $\alpha+\alpha_{k}$ we can add two simple roots, $\alpha_{k-1}$ and $\alpha_{k+1}$ and get a root. Thus there are no roots at level $n-2$ in $\Lambda$. This implies that if $\alpha, \beta \in \Lambda$ then $\alpha(H)+\beta(H) \geq h$ so the corresponding ideal in $\mathfrak{b}$ is abelian. This completes the proof of Lemma 12 .

It is convenient to continue our discussion of the possible $\Lambda$ using the " $\varepsilon$ " notation for the roots thus we identify $\mathfrak{h}$ with $\mathbb{C}^{n}$ and so $H=(n-1, n-2, \ldots, 1,0)$. The above implies that the only $\alpha \in \Phi^{+}$that can possibly be elements of $\Lambda$ are $\varepsilon_{1}-\varepsilon_{n}$ and $\varepsilon_{i}+\varepsilon_{j}$ for $1 \leq i<j \leq n$. We observe that if $\varepsilon_{i}+\varepsilon_{j} \in \Lambda$ then $\varepsilon_{p}+\varepsilon_{q} \in \Lambda$ for $1 \leq p \leq i<q \leq j$. We first assume that $\varepsilon_{1}-\varepsilon_{n} \in \Lambda$ then $\varepsilon_{1}+\varepsilon_{j} \in \Lambda$ for any $2 \leq j \leq n-1$. Thus there is at most one more root in $\Lambda$. If the root were of the form $\varepsilon_{i}+\varepsilon_{j}, 3 \leq i<j \leq n$ then we must have $\varepsilon_{i-1}+\varepsilon_{j} \in \Lambda$. Similarly if $\varepsilon_{2}+\varepsilon_{j} \in \Lambda$ for $j>3$ then we would have the same problem. So there would be too many roots in $\Lambda$. Thus the element must be of the form $\varepsilon_{1}+\varepsilon_{j}$ or $\varepsilon_{2}+\varepsilon_{3}$. Hence $\Lambda$ can only be one of

$$
\left\{\varepsilon_{1}-\varepsilon_{n}\right\} \cup\left\{\varepsilon_{1}+\varepsilon_{2}, \ldots, \varepsilon_{1}+\varepsilon_{n}\right\}
$$

or

$$
\left\{\varepsilon_{1}-\varepsilon_{n}\right\} \cup\left\{\varepsilon_{1}+\varepsilon_{2}, \ldots, \varepsilon_{1}+\varepsilon_{n-1}\right\} \cup\left\{\varepsilon_{2}+\varepsilon_{3}\right\} .
$$

Clearly, both of these sets satisfy 1 . and 2. The rest of the possible choices for $\Lambda$ involve only the elements $\varepsilon_{i}+\varepsilon_{j}$. Using the above observation these sets must be of the form

$\left\{\varepsilon_{1}+\varepsilon_{2}, \ldots, \varepsilon_{1}+\varepsilon_{m_{1}}\right\} \cup\left\{\varepsilon_{2}+\varepsilon_{3}, \ldots, \varepsilon_{2}+\varepsilon_{m_{2}}\right\} \cup \cdots \cup\left\{\varepsilon_{k}+\varepsilon_{k+1}, \ldots, \varepsilon_{k}+\varepsilon_{m_{k}}\right\}$.

with $m_{1} \geq \ldots \geq m_{k}, m_{i}>i$ and the sum $\sum_{i=1}^{k}\left(m_{i}-i\right)=n$. Again, an easy check says that these sets all satisfy 1 . and 2 . Here we see that the number of such ideals is one more than the number of partitions of $n$ with distinct parts.

$\mathrm{E}_{6}$ : In this case it is convenient to label the positive roots as 6 tuples where we take the simple roots in the Bourbaki order. One checks that the sets $\Lambda$ are given as follows:

$$
\begin{aligned}
& \Lambda_{1}=\{(0,1,1,2,2,1),(1,1,1,2,2,1),(1,1,2,2,1,1),(1,1,2,2,2,1)\} \cup \\
& \{(1,1,2,3,2,1),(1,2,2,3,2,1)\} \text {, } \\
& \Lambda_{2}=\{(1,1,2,2,1,0),(1,1,1,2,2,1),(1,1,2,2,1,1),(1,1,2,2,2,1)\} \cup \\
& \{(1,1,2,3,2,1),(1,2,2,3,2,1)\}, \\
& \Lambda_{3}=\{(1,1,1,2,1,1),(1,1,1,2,2,1),(1,1,2,2,1,1),(1,1,2,2,2,1)\} \cup \\
& \{(1,1,2,3,2,1),(1,2,2,3,2,1)\} \text {. }
\end{aligned}
$$

$\mathrm{E}_{7}$ : For this case the sets $\Lambda$ are given as follows. Set 


$$
\begin{aligned}
& V=\{(1,2,2,3,2,2,1),(1,2,2,3,3,2,1),(1,2,2,4,3,2,1)\} \cup \\
& \quad\{(1,2,3,4,3,2,1),(2,2,3,4,3,2,1)\}
\end{aligned}
$$

$$
\begin{aligned}
& \Lambda_{1}=\{(1,2,2,3,2,1,0),(1,2,2,3,2,1,1)\} \cup V . \\
& \Lambda_{2}=\{(1,1,2,3,2,2,1),(1,1,2,3,3,2,1)\} \cup V \\
& \Lambda_{3}=\{(1,2,2,3,2,1,1),(1,1,2,3,3,2,1)\} \cup V
\end{aligned}
$$

$\mathrm{E}_{8}$ : Here is the list in this case:

If $V$ is given by

$$
\begin{aligned}
& \{(2,3,3,5,4,3,2,1),(2,3,4,5,4,3,2,1),(2,3,4,6,4,3,2,1)\} \cup \\
& \{(2,3,4,6,5,3,2,1),(2,3,4,6,5,4,2,1)\} \cup \\
& \{(2,3,4,6,5,4,3,1),(2,3,4,6,5,4,3,2)\}
\end{aligned}
$$

then we have

$$
\{(1,3,3,5,4,3,2,1)\} \cup V
$$

and

$$
\{(2,2,4,5,4,3,2,1)\} \cup V .
$$

$\mathrm{F}_{4}$ : In this case there is only one choice:

$$
\{(1,2,3,2),(1,2,4,2),(1,3,4,2),(2,3,4,2)\} .
$$

$\mathrm{G}_{2}$ : Here there is only one choice:

$$
\{(3,1),(3,2)\} .
$$

\section{The main result.}

We retain the notation of the previous sections. We will assume that $\mathfrak{g}$ is simple. Let $G_{r}(\mathfrak{g})$ denote the Grassmann variety of $r$-dimensional subspaces of $\mathfrak{g}$ and let $\mathcal{A}_{r}(\mathfrak{g})$ be the closed subvariety of $r$-dimensional Lie subalgebras of $\mathfrak{g}$. In this section we will study the natural action of $G$ on $\mathcal{A}_{r}(\mathfrak{g})$. Fix $B$ a Borel subgroup of $G$ with Lie algebra $\mathfrak{b}$ and $\mathfrak{h} \subset \mathfrak{b}$ a Cartan subalgebra (as in the previous sections).

Lemma 13 Assume that $1 \leq r \leq$ l. If $\mathfrak{a} \in \mathcal{A}_{r}(\mathfrak{g})$ is such that $G \mathfrak{a}$ is closed then there exists a Borel subalgebra $\mathfrak{b}_{1}$ of $\mathfrak{g}$ such that $\mathfrak{a}$ is an ideal in $\mathfrak{b}_{1}$ contained in $\left[\mathfrak{b}_{1}, \mathfrak{b}_{1}\right]$. Furthermore, $\mathfrak{a}$ is abelian. 
Proof. The Borel fixed point theorem implies that $B$ has a fixed point in $G \mathfrak{a}$. Thus by replacing $\mathfrak{a}$ by $g \mathfrak{a}$, if necessary, we may assume that $B \mathfrak{a}=\mathfrak{a}$. Since this implies that $[\mathfrak{h}, \mathfrak{a}] \subset \mathfrak{a}$ we see that $\mathfrak{a}=\mathfrak{h} \cap \mathfrak{a} \oplus \sum_{\alpha \in \Phi} \mathfrak{a} \cap \mathfrak{g}_{\alpha}$. This implies that $\mathfrak{a}$ must contain the highest root. Since $\operatorname{dim} \mathfrak{a}=r \leq l$ this implies that $\mathfrak{a} \subset[\mathfrak{b}, \mathfrak{b}]$ and is an ideal in $\mathfrak{b}$. The case by case argument of the previous section implies that $\mathfrak{a}$ is abelian.

Our main result is

Theorem 14 Let $\mathfrak{a}$ be an ideal in $\mathfrak{b}$ of dimension $l$ then $\mathfrak{a}$ is in the Zariski closure of $B \mathfrak{g}^{e}$.

This result will be proved by a case by case check. The arguments are complicated but are based on a small number of ideas (most of which appear in the first case). We encourage the serious reader to carry out the steps indicated in the arguments for a specific value of $n$ (for the classical groups). We note that the Borel fixed point theorem implies that the closed orbits of $B$ in $\mathcal{A}_{r}(\mathfrak{b})$ are fixed points which are therefore the ideals of $\mathfrak{b}$. Thus there is at least one such element in the Zariski closure of $B \mathfrak{g}^{e}$. This combined with the observations for $\mathrm{F}_{4}$ and $\mathrm{G}_{2}$ implies the theorem in these two cases. We now begin the analysis of the other cases.

$\mathrm{A}_{n}$ : We set $e_{i}=\sum_{j=1}^{n+1-i} E_{j j+i}$ for $i=1, \ldots, n$. Using the standard imbedding of the Grassmannian, $G_{l}(\mathfrak{b})$ in $\mathbb{P}\left(\wedge^{l} \mathfrak{b}\right)$ we must show that if $\mathfrak{a}$ is an abelian ideal in $\mathfrak{b}$ of dimension $n$ then $\wedge^{n} \mathfrak{a} \in \overline{B\left(e_{1} \wedge \cdots \wedge e_{n}\right)}$. We will set up a combinatorial method to prove the result in this case. Many of the techniques will be used for the other simple types. We will be using the " $\varepsilon$ " notation for roots. We will freely interchange partitions, subsets $\Lambda$ of $\Phi^{+}$satisfying 1 . and 2. of the previous section and ideals in $\mathfrak{b}$ of dimension $l$. We need a bit more notation before we get started. Let $H_{i}=\sum_{j \leq i} E_{i i}-\frac{i}{n+1} I$. We will also use the notation $u_{i}$ for $E_{i i}-\frac{1}{n+1} I$. Then we note that if $p<q$

$$
e^{t a d H_{i}} E_{p q}=\left\{\begin{array}{c}
e^{t} E_{p q}, p \leq i \\
E_{p q}, \text { otherwise }
\end{array} .\right.
$$

The analogous formula for $u_{i}$ is

$$
e^{t a d u_{i}} E_{p q}=\left\{\begin{array}{c}
e^{t} E_{p q}, p=i \\
e^{-t} E_{p q}, q=i \\
E_{p q}, \text { otherwise }
\end{array} .\right.
$$

This implies that

$$
e^{t a d H_{1}} e_{i}=e^{t} E_{1, i+1}+\sum_{j=2}^{n+1-i} E_{j, j+i}
$$

so

$$
e^{-t n} e^{t a d H_{1}}\left(e_{1} \wedge \cdots \wedge e_{n}\right)=E_{12} \wedge E_{13} \wedge \cdots \wedge E_{1, n+1}+O\left(e^{-t}\right)
$$


as $t \rightarrow+\infty$. We will use the notation $[w]$ for $\mathbb{C} w$ in $\mathbb{P}\left(\wedge^{l} \mathfrak{g}\right)$ (the projective space). The displayed formula now implies that

$$
\lim _{t \rightarrow \infty} e^{t a d H_{1}}\left[e_{1} \wedge \cdots \wedge e_{n}\right]=\left[E_{12} \wedge E_{13} \wedge \cdots \wedge E_{1, n+1}\right]
$$

This proves the result for the $\mathfrak{a}$ corresponding to the partition $n$. For the conjugate partition (a column of $n, 1$ 's)

$$
\Lambda=\left\{\varepsilon_{1}-\varepsilon_{n+1}, \varepsilon_{2}-\varepsilon_{n+1}, \ldots, \varepsilon_{n}-\varepsilon_{n+1}\right\} .
$$

Using $H_{n}$ we find

$$
e^{-t n} e^{t a d H_{n}}\left(e_{1} \wedge \cdots \wedge e_{n}\right)=E_{n, n+1} \wedge E_{2, n+1} \wedge \cdots \wedge E_{1, n+1}+O\left(e^{-t}\right) .
$$

Proving the result in this case.

Let $p_{1} \geq p_{2} \geq \ldots \geq p_{m}$ be a partition of $n$. We will prove that the corresponding ideal is in the closure of $B \wedge^{l} \mathfrak{g}^{e}$. If $m=1$ or $m=n$ then we have proved the result above. We may assume that $1<p_{1}<n$ and $n>m>1$. The ideal in $\mathfrak{b}$ corresponding to this partition considered as an element of $\mathbb{P}\left(\wedge^{l} \mathfrak{g}\right)$ is

$\left[E_{1, n+2-p_{1}} \wedge \cdots \wedge E_{1, n+1} \wedge E_{2, n+2-p_{2}} \wedge \cdots \wedge E_{2, n+1} \wedge \cdots \wedge E_{m, n+2-p_{m}} \wedge \cdots \wedge E_{m, n+1}\right]$.

We will now approach this element in the closure of $B\left[e_{1} \wedge \cdots \wedge e_{n}\right]$ in a sequence of steps. We first show that if $1 \leq k<m$ and $p_{k}>1$ then the element

$$
\begin{aligned}
& {\left[e_{p_{1}+\ldots+p_{k}-k+1} \wedge \cdots \wedge e_{n-k} \wedge E_{1, n+2-p_{1}} \wedge \cdots \wedge E_{1, n+1} \wedge E_{2, n+2-p_{2}} \wedge \cdots\right.} \\
& \left.\wedge E_{2, n+1} \wedge \cdots \wedge E_{k, n+2-p_{k}} \wedge \cdots \wedge E_{k, n+1} \wedge E_{k+1, n+1}\right]
\end{aligned}
$$

is in the closure. We prove this by induction on $k$. We first note that

$$
e^{\frac{1}{t} a d E_{1, n+1-p}} e_{j}=\left\{\begin{array}{c}
e_{j}+\frac{1}{t} E_{1, n+1-(p-j)}, 1 \leq j \leq p \\
e_{j}, p<j \leq n
\end{array}\right.
$$

since $e_{n}=E_{1, n+1}$ and $e_{n-1}=E_{1, n}+E_{2, n+1}$ we see that if $p>1$ then

$$
\begin{gathered}
t^{p-1} e^{\frac{1}{t} a d E_{1, n+1-p}}\left(e_{1} \wedge \cdots \wedge e_{n}\right)= \\
E_{1, n+2-p} \wedge \cdots \wedge E_{1, n} \wedge e_{p} \wedge \cdots \wedge e_{n-2} \wedge E_{1, n+1} \wedge E_{2, n+1}+O(t), t \rightarrow 0 .
\end{gathered}
$$

Thus we have

$\lim _{t->0} e^{\frac{1}{t} a d E_{1, n+1-p}}\left[e_{1} \wedge \cdots \wedge e_{n}\right]=\left[e_{p} \wedge \cdots \wedge e_{n-2} \wedge E_{1, n+2-p} \wedge \cdots \wedge E_{1, n} \wedge E_{1, n+1} \wedge E_{2, n+1}\right]$.

This is the assertion for $k=1$. Assume that we have proved the assertion for $k-1 \geq 1$ and $k<m, p_{k}>1$. Then we know that

$$
\begin{aligned}
u & =\left[e_{p_{1}+\ldots+p_{k-1}-k+2} \wedge \cdots \wedge e_{n-k+1} \wedge E_{1, n+2-p_{1}} \wedge \cdots \wedge E_{1, n+1} \wedge E_{2, n+2-p_{2}} \wedge \cdots\right. \\
& \left.\wedge E_{2, n+1} \wedge \cdots \wedge E_{k-1, n+2-p_{k-1}} \wedge \cdots \wedge E_{k-1, n+1} \wedge E_{k, n+1}\right]
\end{aligned}
$$


is in the closure. We also note that $e_{p_{1}+\ldots+p_{k-1}-k+2}=\sum E_{r, r+p_{1}+\ldots+p_{k-1}-k+2}$ over $1 \leq r \leq n-1+k-p_{1}-\ldots-p_{k-1}$. We consider the term with $r+p_{1}+\ldots+$ $p_{k-1}-k+2=n+2-p_{k}$. That is $r=n-p_{1}-\ldots-p_{k}+k=p_{k+1}+\ldots+p_{m}+k \geq k+1$ since $m>k$. We will now analyze $e^{\frac{1}{t} a d E_{k, r}} u$ as $t \rightarrow 0$. The factors in $u$ that have a term of the form $E_{r, j}$ are $e_{p_{1}+\ldots+p_{k-1}-k+2}, \ldots, e_{p_{1}+\ldots+p_{k-1}-k+1+p_{k}}$ and the terms are $E_{r, n+2-p_{k}}, \ldots, E_{r, n+1}$. As above we have all of the factors in $u$ are fixed by $e^{\frac{1}{t} a d E_{k, r}}$ except for these (we note that every term appearing in the product has second entry at least $k$ ) and for these

$$
e^{\frac{1}{t} a d E_{k, r}} e_{p_{1}+\ldots+p_{k-1}-k+1+j}=e_{p_{1}+\ldots+p_{k-1}-k+1+j}+\frac{1}{t} E_{k, n-p_{k}+1+j} .
$$

Now $E_{k, n+1}$ is already a factor see that $\lim _{t \rightarrow 0} e^{\frac{1}{t} a d E_{k, r}} u$ is

$$
\begin{aligned}
& {\left[e_{p_{1}+\ldots+p_{k}-k+1} \wedge \cdots \wedge e_{n-k} \wedge E_{1, n+2-p_{1}} \wedge \cdots \wedge E_{1, n+1} \wedge E_{2, n+2-p_{2}} \wedge \cdots\right.} \\
& \left.\wedge E_{2, n+1} \wedge \cdots \wedge E_{k, n+2-p_{k}} \wedge \cdots \wedge E_{k, n+1} \wedge E_{k+1, n+1}\right] .
\end{aligned}
$$

This completes the induction. We can now prove the theorem in the case of $A_{n}$.

If $m=1$ all of the $p_{i}$ are 1 then we are done. We may thus assume that $m \geq 2$ and either $p_{1} \geq \ldots \geq p_{m}>1$ or we have $p_{1} \geq \ldots \geq p_{k}>1$ and $p_{k+1}=\ldots=p_{m}=1$. We apply our assertion above for $k=m-1$. We then have

$$
\begin{aligned}
v & =\left[e_{p_{1}+\ldots+p_{m-1}-m+2} \wedge \cdots \wedge e_{n-m+1} \wedge E_{1, n+2-p_{1}} \wedge \cdots \wedge E_{1, n+1} \wedge E_{2, n+2-p_{2}} \wedge \cdots\right. \\
& \left.\wedge E_{2, n+1} \wedge \cdots \wedge E_{m-1, n+2-p_{k}} \wedge \cdots \wedge E_{m-1, n+1} \wedge E_{m, n+1}\right]
\end{aligned}
$$

in the closure. We note that $m+p_{1}+\ldots+p_{m-1}-m+2=n+2-p_{m}$. Thus we have in the first case $\lim _{t \rightarrow+\infty} e^{t a d H_{m}} v=$

$$
\left[E_{1, n+2-p_{1}} \wedge \cdots \wedge E_{1, n+1} \wedge E_{2, n+2-p_{2}} \wedge \cdots \wedge E_{2, n+1} \wedge \cdots \wedge E_{m, n+2-p_{m}} \wedge \cdots \wedge E_{m, n+1}\right]
$$

as desired. In the second case we note that if $k=m-1$ we are already done. If not we argue as in the beginning of the proof to see that

$$
\lim _{t \rightarrow \infty} e^{t H_{n}} v
$$

is the desired element. Since we have covered both cases the proof for $A_{n}$ is complete.

$\mathrm{B}_{n}, n \geq 2$. We use the realization in section 2 . We will do this case in a great deal of detail and just show how one modifies the methods for $C_{n}$ and $D_{n}$. We note that if $n=2$ then there is only one such ideal so we may assume that $n \geq 3$. We assume that the reader has looked at the methods for the previous case. We write

$$
\begin{gathered}
X_{\varepsilon_{j}}=E_{j, n+1}-E_{n+1, n+1+j}, \\
X_{\varepsilon_{i}-\varepsilon_{j}}=E_{i j}-E_{2 n+2-j, 2 n+2-i}, i<j,
\end{gathered}
$$




$$
X_{\varepsilon_{i}+\varepsilon_{j}}=E_{i, 2 n+2-j}-E_{j, 2 n+2-i}, i<j .
$$

The subsets $\Lambda$ under consideration are

$$
\left\{\varepsilon_{1}+\varepsilon_{2}, \varepsilon_{1}+\varepsilon_{3}, \ldots, \varepsilon_{1}+\varepsilon_{n}, \varepsilon_{1}\right\}
$$

and

$$
\left\{\varepsilon_{1}+\varepsilon_{2}, \ldots, \varepsilon_{1}+\varepsilon_{m_{1}}\right\} \cup\left\{\varepsilon_{2}+\varepsilon_{3}, \ldots, \varepsilon_{2}+\varepsilon_{m_{2}}\right\} \cup \cdots \cup\left\{\varepsilon_{k}+\varepsilon_{k+1}, \ldots, \varepsilon_{k}+\varepsilon_{m_{k}}\right\}
$$

for $m_{1} \geq m_{2} \geq \ldots \geq m_{k}$ with $m_{i}>i$ and $\sum_{i}\left(m_{i}-i\right)=n$.

We will also use the notation $u_{j}$ for the element of $\mathfrak{h}$ such that $\varepsilon_{i}\left(u_{j}\right)=\delta_{i j}$.

We must show that if $\mathfrak{a}_{\Lambda}$ is the ideal corresponding to $\Lambda$ then it is in the closure of $B \mathfrak{g}^{e}$. We first look at the first of the sets corresponding to ideals in $\mathfrak{b}$. If $n=2 k$ then

$$
\begin{gathered}
v_{0}=\lim _{t \rightarrow+\infty} e^{t a d u_{1}}\left[e_{1} \wedge \cdots \wedge e_{n}\right]= \\
{\left[X_{\varepsilon_{1}-\varepsilon_{2}} \wedge \cdots \wedge X_{\varepsilon_{1}-\varepsilon_{2 k}} \wedge X_{\varepsilon_{1}+\varepsilon_{2 k}} \wedge X_{\varepsilon_{1}+\varepsilon_{2 k-2}} \wedge \cdots \wedge X_{\varepsilon_{1}+\varepsilon_{2}}\right] .}
\end{gathered}
$$

Now

$v_{1}=\lim _{t \rightarrow 0} e^{\frac{1}{t} a d X_{\varepsilon_{2}+\varepsilon_{3}}} v_{0}=\left[X_{\varepsilon_{1}+\varepsilon_{3}} \wedge X_{\varepsilon_{1}+\varepsilon_{4}} \wedge \cdots \wedge X_{\varepsilon_{1}-\varepsilon_{2 k}} \wedge X_{\varepsilon_{1}+\varepsilon_{2 k}} \wedge X_{\varepsilon_{1}+\varepsilon_{2 k-2}} \wedge \cdots \wedge X_{\varepsilon_{1}+\varepsilon_{2}}\right]$

and if $v_{j}$ has been defined with $j<k-1$ then

$$
v_{j+1}=\lim _{t \rightarrow 0} e^{\frac{1}{t} a d X_{\varepsilon_{2 j+3}+\varepsilon_{2 j-2}}} v_{j} .
$$

We have (recall $n=2 k$ )

$$
v_{k}=\left[X_{\varepsilon_{1}-\varepsilon_{2 k}} \wedge X_{\varepsilon_{1}+\varepsilon_{2 k}} \wedge X_{\varepsilon_{1}+\varepsilon_{2 k-1}} \wedge \cdots \wedge X_{\varepsilon_{1}+\varepsilon_{3}} \wedge X_{\varepsilon_{1}+\varepsilon_{2}}\right]
$$

finally

$$
\lim _{t \rightarrow 0} e^{\frac{1}{t} a d X_{n}} v_{k}
$$

is the desired element.

The case when $n=2 k+1$ is almost the same define $v_{0}, \ldots, v_{k}$ in exactly the same way then $v_{k}$ is the desired element.

For the rest of the argument in this case it will be convenient to use the notation

$$
X_{(i, 0)}=X_{\varepsilon_{i}}, X_{(i,-j)}=X_{\varepsilon_{i}-\varepsilon_{j}}, i<j \text { and } X_{(i, j)}=X_{\varepsilon_{i}+\varepsilon_{j}} .
$$

Before we continue we will give another description of the sets $\Lambda \subset \Phi^{+}$that satisfy condition 2 . in section 3 . We note that the set must contain a maximal subset of the form $\Lambda^{(r)}=\left\{\varepsilon_{i}+\varepsilon_{j} \mid 1 \leq i \leq r\right.$ and $\left.i+1 \leq j \leq r+1\right\}$. If $\Lambda \neq \Lambda^{(r)}$ then the rest of $\Lambda$ is given as $\cup_{j=1}^{m_{1}-r-1}\left\{\varepsilon_{i}+\varepsilon_{j+r+1} \mid 1 \leq i \leq p_{j}\right\}$ and

$$
r \geq p_{1} \geq p_{2} \geq \ldots \geq p_{m_{1}-r-1} \geq 0
$$


satisfies $\sum_{j} p_{j}=|\Lambda|-\frac{r(r+1)}{2}$. To see this decomposition we give an example In the array below the first row incicates the elements $\varepsilon_{1}+\varepsilon_{i}$ in $\Lambda$ second indicates the elements $\varepsilon_{2}+\varepsilon_{i}$ etc.

$\begin{array}{lllllll}2 & 3 & 4 & 5 & 6 & 7 & 8 \\ & 3 & 4 & 5 & 6 & 7 & 8 \\ & & 4 & 5 & 6 & 7 & \\ & & & 5 & 6 & & \\ & & & & 6 & & \end{array}$

So here $m_{1}=8, m_{2}=8 . m_{3}=7, m_{4}=6, m_{5}=6, r=5$ and $\Lambda^{(5)}$ corresponds to

$$
\begin{array}{lllll}
2 & 3 & 4 & 5 & 6 \\
& 3 & 4 & 5 & 6 \\
& & 4 & 5 & 6 \\
& & & 5 & 6 \\
& & & & 6
\end{array}
$$

and the rest is

$$
\left\{\varepsilon_{1}+\varepsilon_{7}, \varepsilon_{2}+\varepsilon_{7}, \varepsilon_{3}+\varepsilon_{7}\right\} \cup\left\{\varepsilon_{1}+\varepsilon_{8}, \varepsilon_{2}+\varepsilon_{87}\right\} .
$$

So in this case $p_{1}=3, p_{2}=2, p_{3}=0$. Our strategy in the proof is to use this decomposition of the set and handle the two parts individually.

We prove by induction on $r$ that if $\mathfrak{a}^{(r)}$ is the ideal corresponding to $\Lambda^{(r)}$ then if $r$ is odd the the subspace corresponding to

$$
\wedge^{\left(\begin{array}{c}
r+1 \\
2
\end{array}\right)} \mathfrak{a}^{(r)} \wedge e_{\left(\begin{array}{c}
r+1 \\
2
\end{array}\right)+1-\frac{r+1}{2}} \wedge \cdots \wedge e_{n-\frac{r+1}{2}}
$$

is in the closure of $B \mathfrak{g}^{e}$ and if $r$ is even and if $n>\left(\begin{array}{c}r+1 \\ 2\end{array}\right)$ then the subsubspace corresponding to

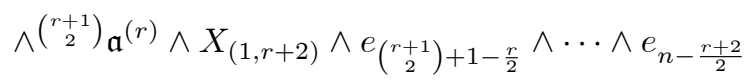

is in the closure of $B \mathfrak{g}^{e}$ or if $r$ is even and $n=\left(\begin{array}{c}r+1 \\ 2\end{array}\right)$ then $\mathfrak{a}^{(r)}$ is in the closure of $B \mathfrak{g}^{e}$. The latter case (with the extra $X_{(1, r+2)}$ will be called a "carry". This is because $e_{n-\frac{r}{2}} \wedge \wedge\left(^{\left(\begin{array}{c}+1 \\ 2\end{array}\right)} \mathfrak{a}^{(r)}=X_{(1, r+2)} \wedge \wedge^{\left(\begin{array}{c}r+1 \\ 2\end{array}\right)} \mathfrak{a}^{(r)}\right.$.

If $r=1$ then since $e_{n}$ is a multiple of $X_{(1,2)}$ and $\mathfrak{a}^{(1)}=\mathbb{C} X_{(1,2)}$ the assertion is that $\mathfrak{g}^{e}$ is in the closure of $B \mathfrak{g}^{e}$. If $r=2$ then the case of equality is $n=3$. In this case

$$
\lim _{t \rightarrow 0} e^{\frac{1}{t} a d X_{23}}\left[e_{1} \wedge e_{2} \wedge e_{3}\right]=\left[e_{2} \wedge X_{(1,3)} \wedge X_{(1,2)}\right]
$$

and $e_{2}$ is up to nonzero multiple $X_{(1,0)}+c X_{(2,3)}$ with $c \neq 0$. Thus

$$
\lim _{t \rightarrow+\infty} e^{t a d u_{2}}\left[e_{2} \wedge X_{(1,3)} \wedge X_{(1,2)}\right]=\wedge^{3} \mathfrak{a}^{(2)}
$$


Note that this completes the proof of the main result for $n=3$. If $n>3$ then

$$
v=\lim _{t \rightarrow 0} e^{\frac{1}{t} a d X_{(2,3)}}\left[e_{1} \wedge e_{2} \wedge \cdots \wedge e_{n}\right]=\left[e_{2} \wedge \cdots \wedge e_{n-1} \wedge X_{(1,3)} \wedge X_{(1,2)}\right] .
$$

If $n=4$ then

$$
\lim _{t \rightarrow 0} e^{\frac{1}{t} a d X_{3}} v=\left[X_{(1,4)} \wedge X_{(1,3)} \wedge X_{(1,2)} \wedge X_{(2,3)}\right]
$$

proving the assertion and completing the proof for $n=4$. If $n>4$ then

$$
\lim _{t \rightarrow 0} e^{\frac{1}{t} a d X_{(3,5)}} v=\left[\left(X_{(1,3)} \wedge X_{(1,2)} \wedge X_{(2,3)}\right) \wedge X_{(1,4)} \wedge e_{3} \wedge \cdots e_{n-2}\right] .
$$

This completes the initiation of the induction and the proof of the main theorem for $n \leq 5$ we can thus assume that $n \geq 6$.

To go on with the induction we will need a bit more notation. We set

$$
\phi_{n}(r)=\left\{\begin{array}{c}
-r \text { if } 1 \leq r \leq n \\
0 \text { if } r=n+1 \\
2 n+2-r \text { if } n+2 \leq r \leq 2 n
\end{array} .\right.
$$

We consider the array, $A$, with $a_{i, j}=\left(i, \phi_{n}(2 j+i-1)\right)$ for $1 \leq i \leq n-j+1$ and $1 \leq j \leq n-i+1$. For example, if $n=9$

$$
\begin{array}{ccccccccc}
(1,-2) & (1,-4) & (1,-6)) & (1,-8) & (1,0) & (1,8) & (1,6) & (1,4) & (1,2) \\
(2,-3) & (2,-5) & (2,-7) & (2,-9) & (2,9) & (2,7) & (2,5) & (2,3) & \\
(3,-4) & (3,-6) & (3,-8) & (3,0) & (3,8) & (3,6) & (3,4) & & \\
& (4 .-5) & (4,-7) & (4,-9) & (4,9) & (4,7) & (4,5) & & \\
A= & (5,-6) & (5,-8) & (5,0) & (5,8) & (5,6) & & & \\
& (6,-7) & (6,-9) & (6,9) & (6,7) & & & & \\
& (7,-8) & (7,0)) & (7,8) & & & & & \\
& (8,-9) & (8,9) & & & & & & \\
(9,0) & & & & & & & &
\end{array}
$$

If we write $\varepsilon(i, m)=\varepsilon_{i}+\operatorname{sgn}(m) \varepsilon_{|m|} . \quad($ defining $\operatorname{sgn}(0)=0)$. Then the $j^{t h}$ column of $A$ is the support of $e_{j}$. We will illustrate the method for this case. Looking at the array we see that

$$
\lim _{t \rightarrow 0} e^{\frac{1}{t} a d X_{(1,4)}}\left[e_{1} \wedge \cdots \wedge e_{9}\right]=\left[X_{(1 ., 2)} \wedge X_{(1,3)} \wedge e_{2} \wedge \cdots \wedge e_{8}\right] .
$$

If we do the same operation using $X_{(2,6)}$ to the outcome we have

$$
\begin{gathered}
{\left[X_{(1,2)} \wedge X_{(1,3)} \wedge X_{(2,3)} \wedge e_{3} \wedge \cdots \wedge e_{8}\right]=\left[X_{(1 ., 2)} \wedge X_{(1,3)} \wedge X_{(1,4)} \wedge X_{(2,3)} \wedge e_{3} \wedge \cdots \wedge e_{7}\right]=} \\
\wedge^{3} \mathfrak{a}^{(2)} \wedge X_{(1,4)} \wedge e_{3} \wedge e_{4} \wedge e_{5} \wedge e_{6} \wedge e_{7} .
\end{gathered}
$$

We now "add" the third row of $\mathfrak{a}^{(3)}$. We "need" $(2,4)$ and $(3,4)$. We use $X_{(2,9)}$ (as above) getting

$$
\wedge^{3} \mathfrak{a}^{(2)} \wedge X_{(1,4)} \wedge X_{(2,4)} \wedge e_{4} \wedge e_{5} \wedge e_{6} \wedge e_{7}
$$


and then $X_{(3,-9)}$ to get

$$
\wedge^{3} \mathfrak{a}^{(2)} \wedge X_{(1,4)} \wedge X_{(2,4)} \wedge X_{(3,4)} \wedge e_{5} \wedge e_{6} \wedge e_{7}=\wedge^{6} \mathfrak{a}^{(3)} \wedge e_{5} \wedge e_{6} \wedge e_{7}
$$

We now assume that we have proved the assertion for $r-1$. We first look at the case when $\left(\begin{array}{c}r+1 \\ 2\end{array}\right)=n$. If $r$ is odd then $r-1$ is even hence the inductive

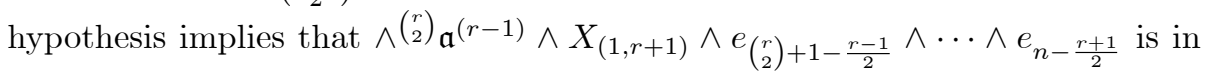
the closure of $B \mathfrak{g}^{e}$. We note that under our hypothesis we have

$$
\left(\begin{array}{l}
r \\
2
\end{array}\right)=n-r .
$$

Thus if $j=\left(\begin{array}{l}r \\ 2\end{array}\right)+1-\frac{r-1}{2}$ then $\phi_{n}(2 j+(r+1)-1)=\phi_{n}(2(n-r+1)-r+1+r)=$ $\phi_{n}(2 n-2 r+3)$. If $2 n-2 r+3 \leq n+1$ then we would have $\left(\begin{array}{c}r+1 \\ 2\end{array}\right) \leq 2 r-2$ and this is not possible. Thus $\phi_{n}(2 n-2 r+3)=2 r-1$. We also note that the element in the third row and the $n-\frac{r+1}{2}$ column is $(3, r+1)$. This implies that the subspace corresponding to

$$
\begin{gathered}
\lim _{t \rightarrow+\infty} e^{t a d u_{r+1}} \wedge\left(_{\left(\begin{array}{c}
r \\
2
\end{array}\right)} \mathfrak{a}^{(r-1)} \wedge X_{(1, r+1)} \wedge e_{\left(\begin{array}{c}
r \\
2
\end{array}\right)+1-\frac{r-1}{2}} \wedge \cdots \wedge e_{n-\frac{r+1}{2}}=\right. \\
\wedge\left(\begin{array}{c}
r \\
2
\end{array}\right) \mathfrak{a}^{(r-1)} \wedge X_{(1, r+1)} \wedge X_{(r+1,2 r-1)} \wedge X_{(r+1,2 r-3)} \wedge \cdots \wedge X_{(r+1, r+2)} \wedge \\
X_{(r, r+1)} \wedge X_{(r-2, r+1)} \wedge \cdots \wedge X_{(3, r+1)} .
\end{gathered}
$$

Now do in order the limits as $t \rightarrow 0$ of $e^{\frac{1}{t} a d X_{(2,-2 r+1)}}, e^{\frac{1}{t} a d X_{(4,-2 r+3)}}, \ldots, e^{\frac{1}{t} a d X_{(r-1,-r-2)}}$ (here the limit of the left most term is taken first) and we have $\wedge\left(\begin{array}{c}r+1 \\ 2\end{array}\right) \mathfrak{a}^{(r)}$ as desired.

If $r$ is even and $\left(\begin{array}{c}r+1 \\ 2\end{array}\right)=n$. then the inductive hypothesis implies that the subspace corresponding to

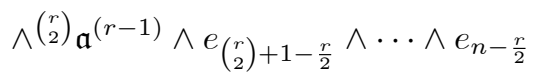

is in the closure of $B \mathfrak{g}^{e}$. If we set $j=\left(\begin{array}{l}r \\ 2\end{array}\right)+1-\frac{r}{2}$ then we see that if $2 j+r \leq n+1$ then $r \leq 2$ but then $n=3$ and the result has been proved in this case. Thus $\phi_{n}(2 j+(r+1)-1)=\phi_{n}(2 n-2 r+2)=2 r$. This time the element in the second row and the $n-\frac{r}{2}$ column is $(2, r+1)$. This implies that

$$
\begin{gathered}
\lim _{t \rightarrow+\infty} e^{t a d u_{r+1}} \wedge\left(\begin{array}{c}
r \\
2
\end{array}\right) \mathfrak{a}^{(r-1)} \wedge e_{\left(\begin{array}{c}
r \\
2
\end{array}\right)+1-\frac{r}{2}} \wedge \cdots \wedge e_{n-\frac{r}{2}}= \\
\wedge\left(\begin{array}{c}
r \\
2
\end{array}\right) \mathfrak{a}^{(r-1)} \wedge X_{(r+1,2 r)} \wedge X_{(r+1,2 r-2)} \wedge \cdots \wedge X_{(r+1, r+2)} \wedge \\
X_{(r, r+1)} \wedge X_{(r-2, r+1)} \wedge \cdots \wedge X_{(2, r+1)} .
\end{gathered}
$$

Now argue in the same way as the previous case.

We are now ready to do the general inductive step. We first look at the case when $n$ is sufficiently large compared with $\left(\begin{array}{c}r+1 \\ 2\end{array}\right)$. For us this will mean that if $r$ 
is odd then $n \geq\left(\begin{array}{c}r+1 \\ 2\end{array}\right)+\frac{r-1}{2}+1$ or if $r$ is even $n \geq\left(\begin{array}{c}r+1 \\ 2\end{array}\right)+\frac{r}{2}+1$. Under this condition we have that if $r$ is odd and $m=\left(\begin{array}{c}r \\ 2\end{array}\right)-\frac{r-1}{2}$ then $n-r+1 \geq m+r-1$. This implies that we can apply in order (left to right)

$$
\lim _{t \rightarrow 0} e^{\frac{1}{t} a d X_{\left(2, \phi_{n}(2(m+1)+r)\right)}}, \lim _{t \rightarrow 0} e^{\frac{1}{t} a d X_{\left(2, \phi_{n}(2(m+2)+r)\right)}}, \ldots, \lim _{t \rightarrow 0} e^{\frac{1}{t} a d X_{\left(r, \phi_{n}(2(m+r-1)+r)\right)}}
$$

to get the desired element. If $r$ is even and if $m=\left(\begin{array}{l}r \\ 2\end{array}\right)-\frac{r}{2}$ then our hypothesis implies that $n-r+1 \geq m+r$ and so we can apply

$$
\lim _{t \rightarrow 0} e^{\frac{1}{t} a d X_{\left(1, \phi_{n}(2(m+1)+r)\right)}}, \lim _{t \rightarrow 0} e^{\frac{1}{t} a d X_{\left(2, \phi_{n}(2(m+2)+r)\right)}}, \ldots, \lim _{t \rightarrow 0} e^{\frac{1}{t} a d X_{\left(r, \phi_{n}(2(m+r)+r)\right)}}
$$

we note that after the second limit we have

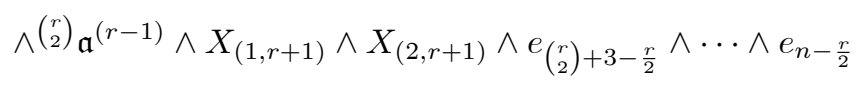

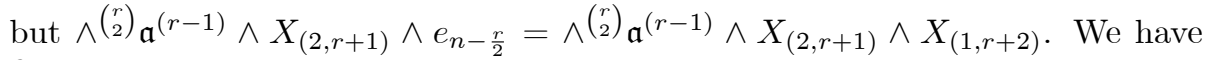
finally completed this induction.

To complete the proof we recall that if $\Lambda$ satisfies condition 2 in section 3 then it is of the form

$$
\Lambda^{(r)} \cup C_{r+1, p_{1}} \cup \cdots \cup C_{m_{1-1}}, p_{m_{1}-r-1}
$$

with $C_{k, p}=\left\{\varepsilon_{i}+\varepsilon_{k+1} \mid i=1, \ldots, p\right\}$ (as above). Let $s$ be the maximum of the even $i$ such that $p_{i} \geq 2, p_{i-1} \geq 3, \ldots$ If no such exists we set $s=0$. We set $X_{C}=X_{\left(a_{1}, b_{1}\right)} \wedge \cdots \wedge X_{\left(a_{s}, b_{s}\right)}$ if $C=\left\{\left(a_{1}, b_{1}\right), \ldots,\left(a_{s}, b_{s}\right\}\right\}$. We argue as above that if $i$ is odd and $r$ is odd then if $i<s$ then the subspace corresponding to

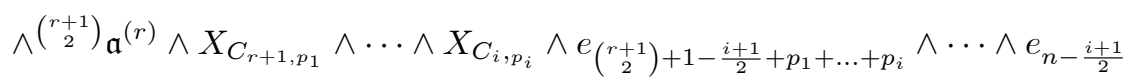

is in the closure of $B \mathfrak{g}^{e}$, if $i>s$ then the subspace corresponding to

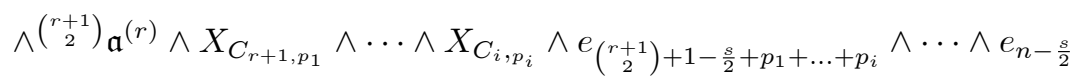

is in the closure, if $i$ is even, $i<m_{1}-1$ and $i \leq s$ then there is a carry that is the subspace corresponding to

$$
\begin{gathered}
\left.\wedge \wedge_{2}^{(r+1}\right) \mathfrak{a}^{(r)} \wedge X_{C_{r+1, p_{1}}} \wedge \cdots \wedge X_{C_{i}, p_{i}} \wedge X_{(1, i+1)} \wedge \\
e_{\left(\begin{array}{c}
r+1 \\
2
\end{array}\right)+1-\frac{i}{2}-p_{1}-\ldots-p_{r}} \wedge \cdots \wedge e_{n-\frac{i+2}{2}}
\end{gathered}
$$

is in the closure of $B \mathfrak{g}^{e}$ otherwise there is no carry and

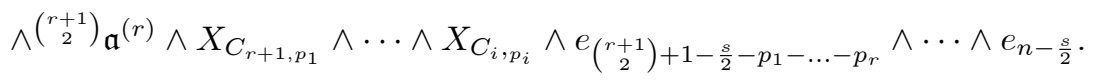

This (finally) implies the result.

We illustrate this with the example $n=9, r=3$ and $p_{1}=2, p_{2}=1$. We have $s=4$ and

$$
\wedge^{6} \mathfrak{a}^{(3)} \wedge e_{5} \wedge e_{6} \wedge e_{7}
$$


is in the closure. Apply $X_{(1,-6)}$ and get

$$
\wedge^{6} \mathfrak{a}^{(3)} \wedge X_{(1,5)} \wedge e_{6} \wedge e_{7}
$$

to this apply $\left.X_{(1,-4)}\right)$ and get (the carry)

$$
\wedge^{6} \mathfrak{a}^{(3)} \wedge X_{(1,5)} \wedge X_{(2,5)} \wedge X_{(1,6)} .
$$

$\mathrm{C}_{n}, n \geq 3$. We will use the " $\varepsilon$ " notation for the roots. We will write $\varepsilon(i, j)=\varepsilon_{i}+\operatorname{sgn}(j) \varepsilon_{|j|}$. Here the positive roots are $\varepsilon_{i}-\varepsilon_{j}$ for $i<j$ and $\varepsilon_{i}+\varepsilon_{j}$ for $i \leq j$. Thus $\varepsilon(2,2)=2 \varepsilon_{2}$. As in the previous case we choose a nonzero root vector, $X_{\alpha}$, for each positive root $\alpha$. We will write $X_{(i, j)}=X_{\varepsilon(i, j)}$ and freely interchange the roots and the pairs. For this case we define

$$
\phi_{n}(r)=\left\{\begin{array}{c}
-r, r \leq n \\
2 n+1-r, r>n
\end{array} .\right.
$$

Then the support of $e_{j}$ is the set $\left\{\varepsilon\left(i, \phi_{n}(2 j+i-1)\right) \mid 1 \leq i \leq n+1-j\right\}$. We lay out the support in an array $A$ where the $j$-th column is the support of $e_{j}$. Thus if $n=9$ we have

$$
\begin{array}{rccccccccc}
(1,-2) & (1,-4) & (1,-6)) & (1,-8) & (1,9) & (1,7) & (1,5) & (1,3) & (1,1) \\
(2,-3) & (2,-5) & (2,-7) & (2,-9) & (2,8) & (2,6) & (2,4) & (2,2) & \\
(3,-4) & (3,-6) & (3,-8) & (3,9) & (3,7) & (3,5) & (3,3) & & \\
& (4 .-5) & (4,-7) & (4,-9) & (4,8) & (4,6) & (4,4) & & & \\
& (5,-6) & (5,-8) & (5,9) & (5,7) & (5,5) & & & \\
(6,-7) & (6,-9) & (6,8) & (6,6) & & & & \\
(7,-8) & (7,9)) & (7,7) & & & & & \\
(8,-9) & (8,8) & & & & & & & \\
(9,9) & & & & & & &
\end{array}
$$

As above we define $u_{j} \in \mathfrak{h}$ by $\varepsilon_{i}\left(u_{j}\right)=\delta_{i j}$.

We first look at the set $\Lambda=\left\{2 \varepsilon_{1}, \varepsilon_{1}+\varepsilon_{2}, \ldots, \varepsilon_{1}+\varepsilon_{n}\right\}$. Then if $n=2 k$ or $n=2 k+1$

$$
\begin{gathered}
\lim _{t \rightarrow+\infty} e^{t a d u_{1}}\left[e_{1} \wedge \cdots \wedge e_{n}\right]= \\
{\left[X_{(1,-2)^{\wedge}} \wedge X_{(1,-4)^{\prime}} \wedge \cdots \wedge X_{(1,-2 k)} \wedge X_{\left(1,2 k-(-1)^{n}\right)} \wedge X_{\left(1,2 k-(-1)^{n}-2\right)} \wedge \cdots \wedge X_{(1,3)} \wedge X_{(1,1)} .\right.}
\end{gathered}
$$

If we apply $\lim _{t \rightarrow 0} e^{\frac{1}{t} a d \sum_{j=1}^{k} X_{(2 k, 2 k)}}$ to this we have $\wedge^{n} \mathfrak{a}_{\Lambda}$. For the set $\Lambda$ one can apply the same method of constructing the set $\Lambda$ column by column as in the case of $\mathrm{B}_{n}$ to prove the main theorem in this case. The arguments are a bit simpler. We won't go into the details but will set up for the calculations. In this case if

$$
\Lambda^{(r)}=\{(i, j) \mid i \leq r, j \leq r, i \leq j\}
$$

and $\mathfrak{a}^{(r)}=\mathfrak{a}_{\Lambda^{(r)}}$ then if $\Lambda \subset \Phi^{+}$is the set of roots in an ideal in $\mathfrak{b}$ then $\Lambda=\Lambda^{(r)} \cup\left(\left\{(1, r+1),(2, r+1), \ldots,\left(p_{r+1}, r+1\right)\right\} \cup \cdots \cup\left\{(1, r+k),(2, r+k), \ldots,\left(p_{r+k}, r+k\right)\right\}\right.$ 
with $r \geq p_{r+1} \geq \ldots \geq p_{r+k} \geq 1$. We will show how the procedure works (we will give details only for the case of $n=9$ ) first we consider the $\Lambda^{(r)}$. We have if $n \geq 5$ then

$$
\lim _{t \rightarrow 0} e^{\frac{1}{t} a d X_{(1,3)}}\left[e_{1} \wedge \cdots \wedge e_{n}\right]=\left[e_{2} \wedge \cdots \wedge e_{n-1} \wedge X_{(1,2)} \wedge X_{(1,1)}\right] .
$$

If we apply $\lim _{t \rightarrow 0} e^{\frac{1}{t} a d X_{(2,5)}}$ to this we have

$$
\left[e_{3} \wedge \cdots \wedge e_{n-1} \wedge X_{(1,2)} \wedge X_{(1,1)} \wedge X_{(2,2)}\right]
$$

we note that

$$
\left[e_{n-1} \wedge X_{(1,2)} \wedge X_{(1,1)} \wedge X_{(2,2)}\right]=\left[X_{(1,3)} \wedge X_{(1,2)} \wedge X_{(1,1)} \wedge X_{(2,2)}\right],
$$

a "carry". We thus have

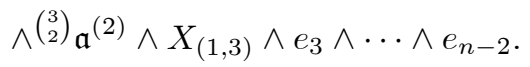

We now assume that $n \geq 9$ then applying $\lim _{t \rightarrow 0} e^{\frac{1}{t} a d X_{(2,8)}}$ we have

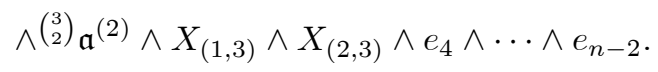

Now we assume that $n=9$ (if $n>9$ then the next step would involve $\lim _{t \rightarrow 0} e^{\frac{1}{t} a d X_{(3,10)}}$ ) we apply $\lim _{t \rightarrow 0} e^{\frac{1}{t} \operatorname{ad} X_{(3,-9)}}$ to this and we get

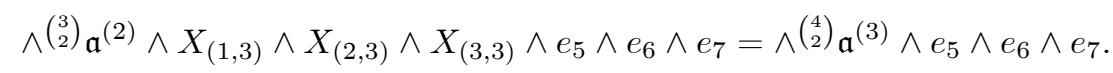

The possible $\Lambda$ containing $\Lambda_{2}$ correspond to $p_{4}=3 ; p_{4}=2, p_{5}=1 ; p_{4}=p_{5}=$ $p_{6}=1$. In the last case we note that

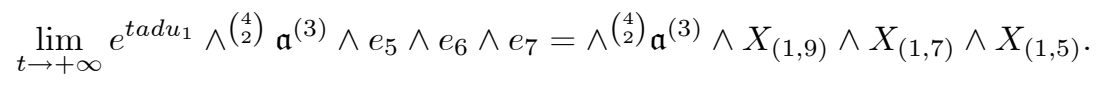

We want the last factor to be $X_{(1,4)} \wedge X_{(1,5)} \wedge X_{(1,6)}$ so apply $\lim _{t \rightarrow 0} e^{\frac{1}{t} a d\left(X_{(4 .,-9)}+X_{(6,-7)}\right.}$ and get the desired element. For the case of $p_{4}=2, p_{5}=1$. We apply $\lim _{t \rightarrow 0} e^{\frac{1}{t} a d X_{(1 .,-8)}}$ to $\wedge^{\left(\begin{array}{l}4 \\ 2\end{array}\right)} \mathfrak{a}^{(3)} \wedge e_{5} \wedge e_{6} \wedge e_{7}$ and get

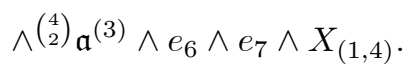

We next apply $\lim _{t \rightarrow 0} e^{\frac{1}{t} \operatorname{ad} X_{(1 .,-6)}}$ and get $\wedge^{\left(\begin{array}{l}4 \\ 2\end{array}\right)} \mathfrak{a}^{(3)} \wedge e_{7} \wedge X_{(1,4)} \wedge X_{(2,4)}$ we note that we have a "carry".

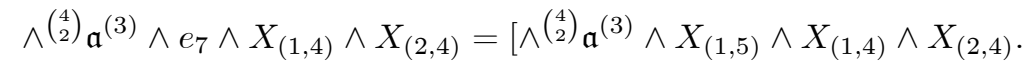

There is one more case $p_{4}=3$. Here we start with $\wedge^{\left(\begin{array}{l}4 \\ 2\end{array}\right)} \mathfrak{a}^{(3)} \wedge e_{6} \wedge e_{7} \wedge X_{(1,4)}$. Now we must avoid the carry so apply $\lim _{t \rightarrow+\infty} e^{t a d u_{4}}$ and get

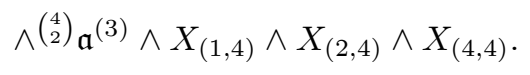


Now apply $\lim _{t \rightarrow 0} e^{\frac{1}{t} a d X_{(3 .,-4)}}$ and get $\wedge\left(\begin{array}{l}4 \\ 2\end{array}\right) \mathfrak{a}^{(3)} \wedge X_{(1,4)} \wedge X_{(2,4)} \wedge X_{(3,4)}$ as desired. We will leave it to the reader to set up the corresponding inductions, carries to prove the result in this case.

$\mathrm{D}_{n}, n \geq 4$. As we shall see, this case is almost identical with that of $\mathrm{B}_{n}$ with one complication (involving the Pfaffian) that we will study now. Here we will use the realization in section 2 . We write (as in the case of $\mathrm{B}_{n}$ )

$$
X_{(i,-j)}=X_{\varepsilon_{i}-\varepsilon_{j}}=E_{i j}-E_{2 n+1-j, 2 n+1-i} \text { for } 1 \leq i<j \leq n
$$

and

$$
X_{(i, j)}=X_{\varepsilon_{i}+\varepsilon_{j}}=E_{i .2 n+1-j}-E_{j, 2 n+1-i} \text { for } 1 \leq i<j \leq n .
$$

If $i>j$ then $X_{(i, j)}=-X_{(j, i)}$. Then $e=\sum_{i=1}^{n-1} X_{\varepsilon_{i}-\varepsilon_{i+1}}+X_{\varepsilon_{n-1}+\varepsilon_{n}}$. With this notation we have

$$
\nabla \operatorname{pfaff}(e)=c_{n}\left(X_{\varepsilon_{1}-\varepsilon_{n}}-X_{\varepsilon_{1}+\varepsilon_{n}}\right) .
$$

Indeed, let $\zeta$ be an outer automorphism of $\mathfrak{g}$ such that $\zeta \varepsilon_{i}=\varepsilon_{i}, i<n$ and $\zeta \varepsilon_{n}=-\varepsilon_{n}$. Then we may assume that $\zeta e=e$. Under this condition we have observed that $\zeta p f a f f=-p f a f f$. We also note (if $A$ is an $m \times m$ matrix that $A_{i j}$ is its $i j$ entry)

Lemma $15\left(e^{n-1}\right)_{1 n}=\left(e^{n-1}\right)_{1 n+1}=1$.

Proof. We write

$$
e=\left[\begin{array}{cc}
J & M \\
0 & -J
\end{array}\right]
$$

with $J$ the standard $n \times n$ nilpotent Jordan block $\sum E_{i, i+1}$ and $M=E_{n-1,1}-$ $E_{n, 2}$. Then

$$
e^{k}=\left[\begin{array}{cc}
J^{k} & M_{k} \\
0 & (-1)^{k} J^{k}
\end{array}\right]
$$

and $M_{k+1}=J M_{k}+(-1)^{k} M J^{k}$. We note that $E_{i, j} J$ is $E_{i, j+1}$ or 0 . Thus only the first term contains $E_{n-k, i}$ for some $i$. This implies that in the expansion of $M_{n-1}$ indicated by the above recursion the only term that has a nonzero entry in the fist row is $J^{n-1} M$. This implies that the first row of $e^{n-1}$ is all 0 's except for $1^{\prime} s$ in the $n$ and $n+1$ position. This proves the lemma.

We use the same notation as in the case of $\mathrm{B}_{n}$. We label the $e_{j}$ so that they are in non-decreasing order by the eigenvalue of $\operatorname{ad}(h)$ and if $n$ is even the gradient of the Pfaffian will have the smaller index. We will also use the notation $u_{i}$ for the element of $\mathfrak{h}$ such that $\varepsilon_{j}\left(u_{i}\right)=\delta_{i j}$.

If $n$ is even $n=2 k$ then up to scalar multiple we have

$$
\begin{gathered}
e_{1} \wedge e_{2} \wedge \cdots \wedge e_{n}=e_{1} \wedge e_{2} \wedge \cdots \wedge e_{k-1} \wedge X_{(1,-n)} \wedge X_{(1, n)} \wedge e_{k+2} \wedge \cdots \wedge e_{n}+ \\
e_{1} \wedge e_{2} \wedge \cdots \wedge e_{k-1} \wedge X_{(1,-n)} \wedge z \wedge e_{k+2} \wedge \cdots \wedge e_{n}-e_{1} \wedge e_{2} \wedge \cdots \wedge e_{k-1} \wedge X_{(1, n)} \wedge z \wedge e_{k+2} \wedge \cdots \wedge e_{n} .
\end{gathered}
$$


With $z=\sum_{i=2}^{k} X_{(i, i+1)}$. Using this we can write out the matrix of supports in this case. This time we set (here $n$ can be even or odd).

$$
\phi_{n}(r)=\left\{\begin{array}{c}
-r, 1 \leq r \leq n \\
2 n+2-r, n+2 \leq r \leq 2 n
\end{array}\right.
$$

we set $a_{i, j}=\left(i, \phi_{n}(2 j+i-1)\right)$ if $j \neq k, k+1,2 j+i-1 \neq n+1, a_{i, j}=(i-1, n)$ if $2 j+i-1=n+1$ and $i+j \leq n+1$. the matrix of supports with the $k$ and $k+1$ column deleted is given by $A=\left(a_{i j}\right)$ thus if $n=10$ then

$$
\begin{array}{ccccccccccc}
(1,-2) & (1,-4) & (1,-6)) & (1,-8) & * & * & (1,8) & (1,6) & (1,4) & (1,2) \\
(2,-3) & (2,-5) & (2,-7) & (2,-9) & * & * & (2,7) & (2,5) & (2,3) & \\
(3,-4) & (3,-6) & (3,-8) & (3,-10) & * & * & (3,6) & (3,4) & & \\
& (4 .-5) & (4,-7) & (4,-9) & (3,10) & * & * & (4,5) & & & \\
A= & (5,-6) & (5,-8) & (5,-10) & (4,9) & * & * & & & \\
& (6,-7) & (6,-9) & (5,10) & (5,8) & * & * & & & \\
& (7,-8) & (7,-10)) & (6,9) & (6,7) & & & & & \\
& (8,-9) & (7,10) & (7,8) & & & & & & \\
(9,-10) & (8,9) & & & & & & & & \\
& (9,10) & & & & & & & & &
\end{array}
$$

The blank columns correspond to $X_{(1,-10)} \wedge X_{(1,10)}+X_{(1,-10)} \wedge z-X_{(1,10)} \wedge z$ with $z=X_{(2,9)}+X_{(3,8)}+X_{(4,7)}+X_{(5,6)}$.

If $n=2 k+1$ then we set for $i+j \leq n+1, a_{i, j}=\left(i, \phi_{n}(2 j+i-1)\right)$ if $j \neq k+1$ and $2 j+i-1 \neq n+1$ if $2 j+i-1=n+1$ then $a_{i j}=(i-1, n)$. If $j=k+1$ then the rules apply except all entries $a_{i j}$ are blank except for $i=1,2$. Thus if $n=9$ then the matrix of supports is given by

$$
\begin{array}{ccccccccc}
(1,-2) & (1,-4) & (1,-6)) & (1,-8) & (1,-9) & (1,8) & (1,6) & (1,4) & (1,2) \\
(2,-3) & (2,-5) & (2,-7) & (2,-9) & (1,9) & (2,7) & (2,5) & (2,3) & \\
(3,-4) & (3,-6) & (3,-8) & (2,9) & * & (3,6) & (3,4) & & \\
(4 .-5) & (4,-7) & (4,-9) & (3,8) & * & (4,5) & & & \\
(5,-6) & (5,-8) & (4,9) & (4,7) & * & & & & \\
(6,-7) & (6,-9) & (5,8) & (5,6) & & & & \\
(7,-8) & (6,9)) & (6,7) & & & & & \\
(8,-9) & (7,8) & & & & & & & \\
(8,9) & & & & & & & &
\end{array}
$$

We now begin the proof of the main theorem for $\mathrm{D}_{n}$. We will assume that the reader is familiar with the techniques used for types A,B and C. Let $\mathfrak{a}$ correspond to

$$
\left\{\varepsilon_{1}-\varepsilon_{n}\right\} \cup\left\{\varepsilon_{1}+\varepsilon_{2}, \ldots, \varepsilon_{1}+\varepsilon_{n}\right\}
$$

we will first prove that it is in the closure of $B \mathfrak{g}^{e}$. There are two cases $n$ even and $n$ odd. We first look at the case when $n=2 k$. We have

$\lim _{t \rightarrow+\infty} e^{\operatorname{tad}\left(u_{1}\right)}\left[e_{1} \wedge \cdots \wedge e_{n}\right]=x=\left[X_{(1,-2)} \wedge X_{(1,-4)} \wedge \cdots \wedge X_{(1,-n)} \wedge X_{(1, n)} \wedge X_{(1, n-2)} \wedge \cdots \wedge X_{(1,2)}\right]$. 
If we successively calculate

$$
\lim _{t \rightarrow 0} e^{\frac{1}{t} a d X_{(2,3)}} x=x_{1}, \lim _{t \rightarrow 0} e^{\frac{1}{t} a d X_{(4,5)}} x_{1}=x_{2}, \ldots, \lim _{t \rightarrow 0} e^{\frac{1}{t} a d X_{(n-2, n-1)}} x_{k-1}=x_{k} .
$$

Then

$$
x_{k}=\mathfrak{a} .
$$

If $n=2 k+1$ then the the first step yields

$$
\begin{gathered}
\lim _{t \rightarrow+\infty} e^{\operatorname{tad}\left(u_{1}\right)}\left[e_{1} \wedge \cdots \wedge e_{n}\right]=x \\
=\left[X_{(1,-2)} \wedge X_{(1,-4)} \wedge \cdots \wedge X_{(1,-n+1)} \wedge\left(X_{(1,-n)}-X_{(1, n)}\right) \wedge X_{(1, n-1)} \wedge \cdots \wedge X_{(1,2)}\right] .
\end{gathered}
$$

If we do the sequence

$$
\lim _{t \rightarrow 0} e^{\frac{1}{t} a d X_{(2,3)}} x=x_{1}, \lim _{t \rightarrow 0} e^{\frac{1}{t} a d X_{(4.5)}} x_{1}=x_{2}, \ldots, \lim _{t \rightarrow 0} e^{\frac{1}{t} \operatorname{ad} X_{(n-1, n)}} x_{k}=x_{k+1} .
$$

Then the last element is the desired one.

Since the outer automorphism $u_{i} \longmapsto u_{i}, i<n$ and $u_{n} \longmapsto-u_{n}$ preserves $\mathfrak{g}^{e}$ and $B$ this implies that to prove the theorem in this case we need only consider the $\Lambda$ of the form

$$
\left\{\varepsilon_{1}+\varepsilon_{2}, \ldots, \varepsilon_{1}+\varepsilon_{m_{1}}\right\} \cup\left\{\varepsilon_{2}+\varepsilon_{3}, \ldots, \varepsilon_{2}+\varepsilon_{m_{2}}\right\} \cup \cdots \cup\left\{\varepsilon_{r}+\varepsilon_{r+1}, \ldots, \varepsilon_{r}+\varepsilon_{m_{r}}\right\} .
$$

with $m_{1} \geq \ldots \geq m_{r}, m_{i}>i$ and the sum $\sum_{i=1}^{r}\left(m_{i}-i\right)=n$. Exactly as in the case of $\mathrm{B}_{n}$.

We will do the case $m_{1}=n$ separately. In this case $\Lambda=\left\{\varepsilon_{1}+\varepsilon_{2}, \ldots, \varepsilon_{1}+\right.$ $\left.\varepsilon_{n}\right\} \cup\left\{\varepsilon_{2}+\varepsilon_{3}\right\}$. We first note that since $n \geq 4$

$$
\lim _{t \rightarrow 0} e^{\frac{1}{t} \operatorname{ad}\left(X_{(1,4)}\right)}\left[e_{1} \wedge \cdots \wedge e_{n}\right]=\left[e_{2} \wedge \cdots \wedge e_{n-1} \wedge X_{(1,3)} \wedge X_{(1,2)}\right] .
$$

If $n=4$ then $e_{2} \wedge e_{3}$ is up to scalar multiple $X_{(1,-4)} \wedge X_{(1,4)}+c\left(X_{(1,-4)}-\right.$ $\left.X_{(1,4)}\right) \wedge X_{(2,3)}$. Thus

$$
\lim _{t \rightarrow+\infty} e^{t a d u_{4}}\left[e_{2} \wedge e_{3} \wedge X_{(1,3)} \wedge X_{(1,2)}\right]=\left[X_{(1,4)} \wedge X_{(1,3)} \wedge X_{(1,2)} \wedge X_{(2,3)}\right]
$$

as desired.

If $n \geq 5$ then $e^{\frac{1}{t} a d X_{35}} e_{2}=e_{2}+c \frac{1}{t} X_{(2,3)}$ and $e^{\frac{1}{t} a d X_{35}} e_{3} \wedge \cdots e_{n-1} \wedge X_{(1,3)} \wedge$ $X_{(1,2)}=e_{3} \wedge \cdots e_{n-1} \wedge X_{(1,3)} \wedge X_{(1,2)}$. Thus

$\lim _{t \rightarrow 0} e^{\frac{1}{t} a d X_{35}}\left[e_{2} \wedge \cdots \wedge e_{n-1} \wedge X_{(1,3)} \wedge X_{(1,2)}\right]=\left[e_{3} \wedge \cdots \wedge e_{n-1} \wedge X_{(1,3)} \wedge X_{(1,2)} \wedge X_{(2,3)}\right]$.

We look at the special case $n=5$. We have (up non-zero scalar) $e_{3}=X_{(1,-5)}-$ $X_{(1,5)}$, and

$$
\left[e_{4} \wedge X_{(1,3)} \wedge X_{(1,2)} \wedge X_{(2,3)}\right]=\left[X_{(1,4)} \wedge X_{(1,3)} \wedge X_{(1,2)} \wedge X_{(2,3)}\right] .
$$


Thus as above

$\lim _{t \rightarrow+\infty} e^{t a d u_{5}}\left[e_{3} \wedge e_{4} \wedge X_{(1,3)} \wedge X_{(1,2)} \wedge X_{(2,3)}\right]=\left[X_{(1,5)} \wedge X_{(1,4)} \wedge X_{(1,3)} \wedge X_{(1,2)} \wedge X_{(2,3)}\right]$.

We now consider $n=2 k, k>2$. Then we have

$$
\begin{gathered}
w=\lim _{t \rightarrow+\infty} e^{t a d u_{1}}\left[e_{3} \wedge \cdots \wedge e_{n-1} \wedge X_{(1,3)} \wedge X_{(1,2)} \wedge X_{(2,3)}\right]= \\
{\left[X_{(1,-6)} \wedge \cdots \wedge X_{(1,-n+2)} \wedge\left(X_{(1,-n)} \wedge X_{(1, n)}+\left(X_{(1,-n)}-X_{(1, n)}\right) \wedge z\right) \wedge X_{(1, n-2)} \wedge \cdots \wedge X_{(1,4)} \wedge X_{(1,3)} \wedge X_{(1,2)} \wedge X_{(2,3)}\right]}
\end{gathered}
$$$$
\text { with } \sup z=\left\{\varepsilon_{2}+\varepsilon_{n-1}, \ldots, \varepsilon_{k}+\varepsilon_{k+1}\right\} \text {. Now take } \lim _{t \rightarrow+\infty} e^{t a d u_{n}} w \text { and get }
$$$$
\left[X_{(1,-6)} \wedge \cdots \wedge X_{(1,-n+2)} \wedge X_{(1,-n)} \wedge X_{(1, n)} \wedge X_{(1, n-2)} \wedge \cdots \wedge X_{(1,4)} \wedge X_{(1,3)} \wedge X_{(1,2)} \wedge X_{(2,3)}\right] .
$$

If we now argue in the first case above we have the desired outcome.

If $n=2 k+1$ with $k \geq 3$ then

$$
\begin{gathered}
w=\lim _{t \rightarrow+\infty} e^{t a d u_{1}}\left[e_{3} \wedge \cdots \wedge e_{n-1} \wedge X_{(1,3)} \wedge X_{(1,2)} \wedge X_{(2,3)}\right]= \\
{\left[X_{(1,-6)} \wedge \cdots \wedge X_{(1,-n+1)} \wedge\left(X_{(1,-n)}-X_{(1, n)}\right) \wedge X_{(1, n-1)} \wedge \cdots \wedge X_{(1,4)} \wedge X_{(1,3)} \wedge X_{(1,2)} \wedge X_{(2,3)}\right]}
\end{gathered}
$$

As above we calculate $\lim _{t \rightarrow+\infty} e^{t a d u_{n}} w$ and use the argument at the beginning of this discussion to "fill in" the elements $X_{i, 2 j+1}$ with $j=2, \ldots, k-1$.

We may now assume that $\Lambda$ is given as above and $m_{1}<n$. We note that $m_{1}>r$.

Before we continue we will use the same description of the sets $\Lambda \subset \Phi^{+}$ that satisfy condition 2 . in section 3 that are still in question as we did in the case of $\mathrm{B}_{n}$. We note that the set must contain $\Lambda^{(r)}=\left\{\varepsilon_{i}+\varepsilon_{j} \mid 1 \leq i \leq r\right.$ and $i+1 \leq j \leq r+1\}$. The rest of $\Lambda$ is given as $\cup_{j=1}^{m_{1}-r-1}\left\{\varepsilon_{i}+\varepsilon_{j+r+1} \mid 1 \leq i \leq p_{j}\right\}$ and

$$
r \geq p_{1} \geq p_{2} \geq \ldots \geq p_{m_{1}-r-1} \geq 0
$$

satisfies $\sum_{j} p_{j}=|\Lambda|-\frac{r(r+1)}{2}$. Our strategy in the proof is, as in the case of $\mathrm{B}_{n}$, to use this decomposition of the set and handle the two parts individually.

Let $n=2 k$ or $2 k+1$ and set $\nu=n-2 k$. We prove by induction on $r$ that if $\mathfrak{a}^{(r)}$ is the ideal corresponding to $\Lambda^{(r)}$ then if $r+1$ is even and if the inequality $\left(\begin{array}{l}r \\ 2\end{array}\right)+1-\frac{r-1}{2} \leq k+\nu \leq\left(\begin{array}{l}r \\ 2\end{array}\right)+\frac{r+1}{2}$ is not satisfied then

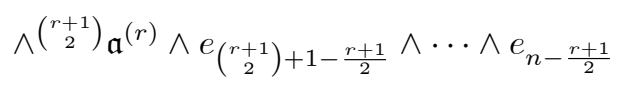

is in the closure of $B \mathfrak{g}^{e}$. If the inequality is satisfied then

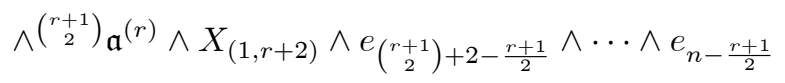

is in the closure of $B \mathfrak{g}^{e}$. If $r+1$ is odd and $n>\left(\begin{array}{c}r+1 \\ 2\end{array}\right)$ then

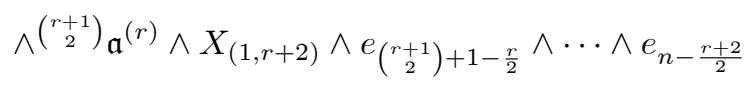


is in the closure of $B \mathfrak{g}^{e}$ or if $n=\left(\begin{array}{c}r+1 \\ 2\end{array}\right)$.then $\mathfrak{a}^{(r)}$ is in the closure.

If $r=1$ then $\mathfrak{a}^{(r)}=\mathbb{C} X_{12}=\mathbb{C} e_{n},\left(\begin{array}{c}r+1 \\ 2\end{array}\right)+1-\frac{r+1}{2}=1$ and $n-\frac{r+1}{2}=n-1$. Thus the assertion in this case is $\mathfrak{g}^{e}$ is in the closure of $\mathfrak{g}^{e}$. If $r=2$ then $\Lambda^{(2)}=\left\{\varepsilon_{1}+\varepsilon_{2}, \varepsilon_{1}+\varepsilon_{3}, \varepsilon_{2}+\varepsilon_{3}\right\}$. We are assuming that $n>3$. As above we have

$$
\lim _{t \rightarrow 0} e^{\frac{1}{t} a d X_{14}}\left[e_{1} \wedge \cdots \wedge e_{n}\right]=\left[X_{(1,2)} \wedge X_{(1,3)} \wedge e_{2} \wedge \cdots \wedge e_{n-1}\right] .
$$

If $n=4$ then as above up to scalar multiple

$$
e_{2} \wedge e_{3}=X_{(1,-4)} \wedge X_{(1,4)}+a X_{(1,-4)} \wedge X_{(2,3)}-a X_{(1,4)} \wedge X_{(2,3)}
$$

with $a \neq 0$ thus

$$
e^{t a d u_{4}} e_{2} \wedge e_{3}=X_{(1,-4)} \wedge X_{(1,4)}+a X_{(1,-4)} \wedge X_{(2,3)}-a X_{(1,4)} \wedge X_{(2,3)}
$$

hence

$$
\lim _{t \rightarrow \infty} e^{t a d u_{4}}\left[X_{(1,2)} \wedge X_{(1,3)} \wedge e_{2} \wedge \cdots \wedge e_{n-1}\right]=\left[X_{(1,2)} \wedge X_{(1,3)} \wedge X_{(1,4)} \wedge X_{(2,3)}\right]
$$

as asserted for this case. We also note that this completes the proof for the special case of $n=4$. If $n>4$ then one checks that

$$
\lim _{t \rightarrow 0} e^{\frac{1}{t} a d X_{24}}\left[X_{(1,2)} \wedge X_{(1,3)} \wedge X_{(2,3)} \wedge X_{(1,4)} \wedge e_{3} \wedge \cdots \wedge e_{n-2}\right]
$$

as asserted. We now begin the inductive step. So we assume the result for $r-1 \geq 3$ and we prove it for $r$. We must assume that $n \geq\left(\begin{array}{c}r+1 \\ 2\end{array}\right)$. First we assume that $r+1$ is even. Then $r$ is odd and the inductive hypothesis implies that

$$
\wedge{ }^{\left(\begin{array}{c}
r \\
2
\end{array}\right)} \mathfrak{a}^{(r-1)} \wedge X_{(1, r+1)} \wedge e_{\left(\begin{array}{c}
r \\
2
\end{array}\right)+1-\frac{r-1}{2}} \wedge \cdots \wedge e_{n-\frac{r+1}{2}}
$$

is in the closure of $B \mathfrak{g}^{e}$. If $n=2 k$ or $2 k+1$ and $\left(\begin{array}{l}r \\ 2\end{array}\right)+1-\frac{r-1}{2}>k+1$ then the argument is identical with that in the case of $\mathrm{B}_{n}$. If $\left(\begin{array}{l}r \\ 2\end{array}\right)+1-\frac{r-1}{2}<k-r+1$ if $n$ is even or if $\left(\begin{array}{l}r \\ 2\end{array}\right)+1-\frac{r-1}{2}<k+2-r$ if $n$ is odd then the argument is essentially the same as the previous two cases. Set $\nu=n-2 k$. The new aspect of the argument is if

$$
\left(\begin{array}{l}
r \\
2
\end{array}\right)+1-\frac{r-1}{2} \leq k+\nu \leq\left(\begin{array}{l}
r \\
2
\end{array}\right)+\frac{r+1}{2}
$$

since then the method in the case of $\mathrm{B}_{n}$ involves the gradient of the Pfaffian. We note that under these circumstances these inequalities are inconsistent with $n=\left(\begin{array}{c}r+1 \\ 2\end{array}\right)$ and $n \geq 6$ (we have proved the theorem for $\left.n \leq 5\right)$. Thus $n>\left(\begin{array}{c}r+1 \\ 2\end{array}\right)$. We follow the method until we come to

$$
u=\wedge\left(^{r} \begin{array}{c}
2 \\
2
\end{array} \mathfrak{a}^{(r-1)} \wedge X_{(1, r+1)} \wedge \cdots \wedge X_{(s, r+1)} \wedge e_{k+\nu} \wedge \cdots \wedge e_{n-\frac{r+1}{2}} .\right.
$$

Now

$\lim _{t \rightarrow 0} e^{\frac{1}{t} a d X_{(r+2, n)}} u=\wedge\left(\begin{array}{c}r \\ 2\end{array}\right) \mathfrak{a}^{(r-1)} \wedge X_{(1, r+1)} \wedge \cdots \wedge X_{(s, r+1)} \wedge X_{(1, r+2)} \wedge e_{k+\nu+1} \wedge \cdots \wedge e_{n-\frac{r+1}{2}}$. 
We now continue as before and have

$$
\wedge\left(_{2}^{r+1}\right)_{\mathfrak{a}^{(r)}} \wedge X_{(1, r+2)} \wedge e_{\left(\begin{array}{c}
r+1 \\
2
\end{array}\right)+2-\frac{r+1}{2}} \wedge \cdots \wedge e_{n-\frac{r+1}{2}} .
$$

For the assertion for $r+1$ odd then if on the previous step we had to use the argument above then we can do the inductive step as in the case of $\mathrm{B}_{n}$ without the first limit. If the inequality

$$
\left(\begin{array}{l}
r \\
2
\end{array}\right)+1-\frac{r}{2} \leq k+\nu \leq\left(\begin{array}{l}
r \\
2
\end{array}\right)+\frac{r+2}{2}
$$

is satisfied then the inductive hypothesis implies that

$$
u=\wedge{ }^{\left(\begin{array}{c}
r \\
2
\end{array}\right)} \mathfrak{a}^{(r-1)} \wedge e_{\left(\begin{array}{c}
r \\
2
\end{array}\right)+1-\frac{r}{2}} \wedge \cdots \wedge e_{n-\frac{r}{2}}
$$

is in the closure of $B \mathfrak{g}^{e}$. We first do

$$
\lim _{t \rightarrow 0} e^{\frac{1}{t} a d X_{(r+2, n)}} u
$$

which yields

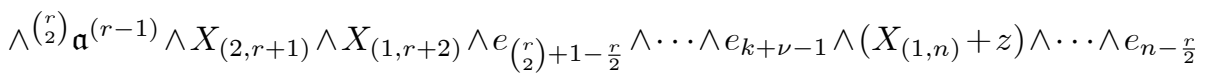

$\ell z$ is at the beginning of this interminable case) if $n$ is even and

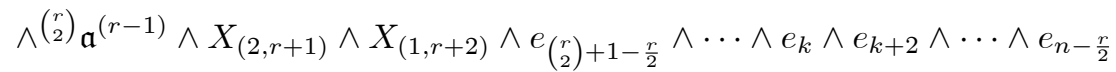

if $n$ is odd. Now continue as in the case of $\mathrm{B}_{n}$.

The hearty reader is encouraged to use the above tables for $n=9$ and 10 to carry out all the techniques in these two cases.

$\mathrm{E}_{8}$ : We must show that the two 8 dimensional ideals in $\mathfrak{b}$ are contained in the closure of $B \mathfrak{g}^{e}$. We will use similar methods for the two examples. Let $V$ be as in the $\mathrm{E}_{8}$ subsection of section 3 . We will choose a non-zero root vector for each positive root. We denote this element by $X(\alpha)$ if $\alpha$ is the root and if say $\alpha=(1,1,1,1,0,0,0,0)$ then we write $X(1,1,1,1,0,0,0,0)$. We write $e_{1}, \ldots, e_{8}$ with $e_{1}=e$ for a basis of $\mathfrak{g}^{e}$ with $\left[H, e_{i}\right]=m_{i} e_{i}$ with $\left(m_{1}, \ldots, m_{8}\right)=$ $(1,7,11,13,17,19,23,29)$. Consider the subset

$$
\begin{gathered}
Z=\{(2,3,4,5,4,3,2,1),(2,3,4,6,4,3,2,1)\} \cup \\
\quad\{(2,3,4,6,5,3,2,1),(2,3,4,6,5,4,2,1)\} \cup \\
\quad\{(2,3,4,6,5,4,3,1),(2,3,4,6,5,4,3,2)\}
\end{gathered}
$$

of $V$. Let $\mathfrak{a}$ be the ideal in $\mathfrak{b}$ that is the sum of these roots spaces. We set $\beta_{1}=(2,3,4,6,5,4,2,1), \beta_{2}=(1,2,3,4,4,3,2,1), \beta_{3}=(1,1,2,3,3,2,2,1), \beta_{4}=$ $(1,1,2,3,2,1,1,1), \beta_{5}=(1,1,2,2,1,0,0,0)$. Then

$$
\lim _{t_{5} \rightarrow 0} e^{\frac{1}{t_{5}} a d X\left(\beta_{5}\right)} \lim _{t_{4} \rightarrow 0} e^{\frac{1}{t_{4}} a d X\left(\beta_{4}\right)} \lim _{t_{3} \rightarrow 0} e^{\frac{1}{t_{3}} a d X\left(\beta_{3}\right)} \lim _{t_{2} \rightarrow 0} e^{\frac{1}{t_{2}} a d X\left(\beta_{2}\right)} \lim _{t_{1} \rightarrow 0} e^{\frac{1}{t_{1}} a d X\left(\beta_{1}\right)}
$$


applied to $\left[e_{1} \wedge \cdots \wedge e_{8}\right]$ yields

$$
\wedge^{6} \mathfrak{a} \wedge e_{6} \wedge e_{7}
$$

Now (taking into account a "carry")

$$
\lim _{t \rightarrow 0} e^{\frac{1}{t} \operatorname{adX}(1,1,1,1,0,0,0,0)} \wedge^{6} \mathfrak{a} \wedge e_{6} \wedge e_{7}
$$

is $\wedge^{8}$ of the ideal corresponding to $V \cup\{(2,2,4,5,4,3,2,1)\}$. For the other ideal we have

$$
\lim _{t \rightarrow 0} e^{\frac{1}{t} \operatorname{ad} X(0,1,1,1,0,0,0,0)} \wedge^{6} \mathfrak{a} \wedge e_{6} \wedge e_{7}=\wedge^{6} \mathfrak{a} \wedge X(1,3,3,5,4,3,2,1) \wedge e_{7} .
$$

Now

$$
\lim _{t \rightarrow+\infty} e^{t a d H_{2}} \wedge^{6} \mathfrak{a} \wedge X(1,3,3,5,4,3,2,1) \wedge e_{7}
$$

yields $\wedge^{8}$ of the ideal corresponding to $\{(1,3,3,5,4,3,2,1)\} \cup V$ and completes the proof of the main result for $\mathrm{E}_{8}$.

$\mathrm{E}_{7}:$ As in the case of $\mathrm{E}_{8}$ we set

$$
\begin{gathered}
Z=\{(1,2,2,3,3,2,1),(1,2,2,4,3,2,1)\} \cup \\
\{(1,2,3,4,3,2,1),(2,2,3,4,3,2,1)\}
\end{gathered}
$$

and we set $\mathfrak{a}$ equal to the ideal corresponding to $Z$. We define $H_{i} \in \mathfrak{h}$ by $\alpha_{j}\left(H_{i}\right)=\delta_{i j}$. Set $\beta_{1}=(1,2,2,4,3,2,1), \beta_{2}=(1,1,2,3,2,1,0), \beta_{3}=(1,1,1,1,1,1,1)$

$$
\begin{gathered}
\lim _{t_{3} \rightarrow 0} e^{\frac{1}{t_{3}} a d X\left(\beta_{3}\right)} \lim _{t_{2} \rightarrow 0} e^{\frac{1}{t_{2}} a d X\left(\beta_{2}\right)} \lim _{t_{1} \rightarrow 0} e^{\frac{1}{t_{1}} a d X\left(\beta_{1}\right)}\left[e_{1} \wedge e_{2} \wedge e_{3} \wedge e_{4} \wedge e_{5} \wedge e_{6} \wedge e_{7}\right]= \\
\wedge^{4} \mathfrak{a} \wedge e_{4} \wedge e_{5} \wedge e_{6} .
\end{gathered}
$$

In order to see the ideal, $\mathfrak{u}$, corresponding to

$$
\Lambda_{1}=\{(1,2,2,3,2,1,0),(1,2,2,3,2,1,1),(1,2,2,3,2,2,1)\} \cup Z
$$

in the closure of $B \mathfrak{g}^{e}$. We set $\gamma_{1}=(1,1,2,2,1,1,1), \gamma_{2}=(1,2,2,3,2,1,0)$ and $\gamma_{1}=(1,2,2,3,2,2,1)$

$$
\lim _{t \rightarrow+\infty} e^{\operatorname{tad}\left(H_{1}+H_{2}+H_{3}\right)} \wedge^{4} \mathfrak{a} \wedge e_{4} \wedge e_{5} \wedge e_{6}=\wedge^{4} \mathfrak{a} \wedge X\left(\gamma_{1}\right) \wedge X\left(\gamma_{2}\right) \wedge X\left(\gamma_{3}\right)
$$

finally

$$
\lim _{t \rightarrow 0} e^{\frac{1}{t} \operatorname{ad} X(0,1,0,1,0,0,0)} \wedge^{4} \mathfrak{a} \wedge X\left(\gamma_{1}\right) \wedge X\left(\gamma_{2}\right) \wedge X\left(\gamma_{3}\right)=\wedge^{7} \mathfrak{u}
$$

The other two cases are

$$
\Lambda_{2}=\{(1,1,2,3,2,2,1),(1,1,2,3,3,2,1),(1,2,2,3,2,2,1)\} \cup Z
$$


and

$$
\Lambda_{3}=\{(1,2,2,3,2,1,1),(1,1,2,3,3,2,1),(1,2,2,3,2,2,1)\} \cup Z .
$$

We have (since there is a "carry")

$$
\begin{gathered}
\lim _{t \rightarrow 0} e^{\frac{1}{t} X(1,0,1,1,1,0,0)} \wedge^{4} \mathfrak{a} \wedge e_{4} \wedge e_{5} \wedge e_{6}= \\
\wedge^{6} \mathfrak{v} \wedge e_{5}
\end{gathered}
$$

with $\mathfrak{v}$ the ideal corresponding to $\{(1,1,2,3,3,2,1),(1,2,2,3,2,2,1)\} \cup Z$. If $\mathfrak{u}_{i}$ corresponds to $\Lambda_{i}$ for $i=2,3$ then

$$
\lim _{t \rightarrow 0} e^{\frac{1}{t} \operatorname{ad} X(0,0,0,1,0,0,0)} \wedge^{6} \mathfrak{v} \wedge e_{5}=\wedge^{7} \mathfrak{u}_{2}
$$

and

$$
\lim _{t \rightarrow 0} e^{\frac{1}{t} a d X(0,1,0,0,0,0,0)} \wedge^{6} \mathfrak{v} \wedge e_{5}=\wedge^{7} \mathfrak{u}_{3} .
$$

This completes the proof for $\mathrm{E}_{7}$.

$\mathrm{E}_{6}$ : In this case we set

$$
\begin{aligned}
& V=\{(1,1,1,2,2,1),(1,1,2,2,1,1),(1,1,2,2,2,1)\} \cup \\
& \quad\{(1,1,2,3,2,1),(1,2,2,3,2,1)\}
\end{aligned}
$$

Then if $\gamma_{1}=(0,1,1,2,2,1), \gamma_{2}=(1,1,2,2,1,0), \gamma_{3}=(1,1,1,2,1,1)$ then the three ideals in question are $\Lambda_{i}=\left\{\gamma_{i}\right\} \cup V$ for $i=1,2,3$. Let $\mathfrak{u}$ be the ideal in $\mathfrak{b}$ corresponding to $V$. Set $\beta_{1}=(1,1,2,3,2,1), \beta_{2}=(1,1,1,1,1,0), \beta_{3}=$ $(0,0,0,1,1,1)$ then

$\lim _{t_{3} \rightarrow 0} e^{\frac{1}{t_{3}} a d X\left(\beta_{3}\right)} \lim _{t_{2} \rightarrow 0} e^{\frac{1}{t_{2}} a d X\left(\beta_{2}\right)} \lim _{t_{1} \rightarrow 0} e^{\frac{1}{t_{1}} a d X\left(\beta_{1}\right)}\left[e_{1} \wedge e_{2} \wedge e_{3} \wedge e_{4} \wedge e_{5} \wedge e_{6}\right]=\wedge^{5} \mathfrak{u} \wedge e_{4}$

we have shown that the support of $e_{4}$ is $\left\{\gamma_{1}, \gamma_{2}, \gamma_{3}\right\}$. Let $\mathfrak{a}_{i}$ be the ideal corresponding to $\Lambda_{i}$. Let $H_{i} \in \mathfrak{h}$ be defined by $\alpha_{j}\left(H_{i}\right)=\delta_{i j}$. Set $v_{1}=$ $H_{2}+H_{3}+H_{4}+H_{5}+H_{6}, v_{2}=H_{1}+H_{2}+H_{3}+H_{4}+H_{5}$ and let $v_{3}=H_{1}+H_{6}$. Then

$$
\lim _{t \rightarrow+\infty} e^{t a d v_{i}} \wedge^{5} \mathfrak{u} \wedge e_{4}=\wedge^{6} \mathfrak{a}_{i} .
$$

This completes the proof of Theorem 14 .

\section{References}

[A] George Andrews, The theory of partitions, Addison-Wesley, Reading, Massachusetts, 1976.

[RB] Ranee Brylinski (Gupta),Abelian algebras and adjoint orbits, Thesis MIT, 1981. 
[K1] Bertram Kostant, The Principal Three-Dimensional Subgroup and the Betti Numbers of a Complex Simple Group, American Journal of Mathematics, 81(1959),973-1032.

[K2] Bertram Kostant, Lie Group Representations on Polynomial Rings, American Journal of Mathematics, 85(1963), 327-404.

[K3] Bertram Kostant, Eigenvalues of a Laplacian and Commutative Lie Subalgebras, Topology, 3(1965), 147-159. 